\title{
Dynamics of Supervised Learning with Restricted Training Sets
}

\author{
A.C.C. Coolen ${ }^{\dagger}$ and D. Saad ${ }^{\ddagger}$
}

$\dagger$ Department of Mathematics, King's College, University of London Strand, London WC2R 2LS, U.K.

$\ddagger$ Department of Computer Science and Applied Mathematics, Aston University Aston Triangle, Birmingham B4 7ET, U.K.

\begin{abstract}
We study the dynamics of supervised learning in layered neural networks, in the regime where the size $p$ of the training set is proportional to the number $N$ of inputs. Here the local fields are no longer described by Gaussian probability distributions. We show how dynamical replica theory can be used to predict the evolution of macroscopic observables, including the relevant performance measures, incorporating the old formalism in the limit $\alpha=p / N \rightarrow \infty$ as a special case. For simplicity we restrict ourselves to single-layer networks and realizable tasks.
\end{abstract}

\section{Contents}

1 Introduction 2

2 From Microscopic to Macroscopic Laws 5

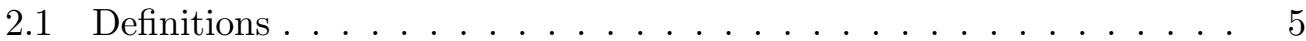

2.2 From Discrete to Continuous Time . . . . . . . . . . . . . . . . 6

2.3 Derivation of Macroscopic Fokker-Planck Equation . . . . . . . . . . 7

$\begin{array}{llr}3 & \text { Application to Canonical Observables } & \mathbf{1 0}\end{array}$

3.1 Choice of Canonical Observables . . . . . . . . . . . . . . . 10

3.2 Deterministic Dynamical Laws . . . . . . . . . . . . . . . . . . . . . 11

3.3 Closure of Macroscopic Dynamical Laws . . . . . . . . . . . . . . . . 14

\begin{tabular}{|lll}
\hline & Replica Calculation of the Green's Function & 16
\end{tabular}

4.1 Disorder Averaging . . . . . . . . . . . . . . . . . . . . . 16

$4.2 \quad$ Derivation of Saddle-Point Equations . . . . . . . . . . . . . . . . . 18

4.3 Explicit Expression for the Green's Function . . . . . . . . . . . . . 21

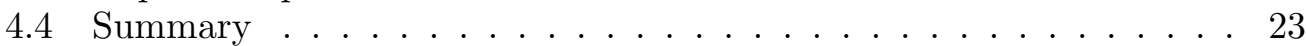

5 Applications and Tests of the Theory 25

5.1 The Limit $\alpha \rightarrow \infty$. . . . . . . . . . . . . . . . 25

5.2 Locally Gaussian Solutions . . . . . . . . . . . . . . . . . . 26

5.3 Explicit Example: Hebbian Learning . . . . . . . . . . . . . . . . . . 29

5.4 Comparison with Exact Results and Simulations . . . . . . . . . . 30

$\begin{array}{lll}6 & \text { Discussion } & 34\end{array}$ 


\section{Introduction}

In the last few years much progress has been made in the analysis of the dynamics of supervised learning in layered neural networks, using the strategy of statistical mechanics: by deriving from the microscopic dynamical equations a set of closed laws describing the evolution of suitably chosen macroscopic observables (dynamic order parameters) in the limit of an infinite system size [e.g. Kinzel and Rujan (1990), Kinouchi and Caticha (1992), Biehl and Schwarze $(1992,1995)$, Saad and Solla (1995)]. A recent review and more extensive guide to the relevant references can be found in Mace and Coolen (1998a). The main successful procedure developed so far is built on the following cornerstones:

- The task to be learned is defined by a (possibly noisy) 'teacher', which is itself a layered neural network. This induces a canonical set of dynamical order parameters, typically the (rescaled) overlaps between the various student weight vectors and the corresponding teacher weight vectors.

- The number of network inputs is (eventually) taken to be infinitely large. This ensures that fluctuations in mean-field observables will vanish and creates the possibility of using the central limit theorem.

- The number of 'hidden' neurons is finite. This prevents the number of order parameters from being infinite, and ensures that the cumulative impact of their fluctuations is insignificant.

- The size of the training set is much larger than the number of updates made. Each example presented is now different from those that have already been seen, such that the local fields will have Gaussian probability distributions, which leads to closure of the dynamic equations.

These are not ingredients to simplify the calculations, but vital conditions, without which the standard method fails. Although the assumption of an infinite system size has been shown not to be too critical (Barber et al, 1996), the other assumptions do place serious restrictions on the degree of realism of the scenarios that can be analyzed, and have thereby, to some extent, prevented the theoretical results from being used by practitioners.

In this paper we study the dynamics of learning in layered neural networks with restricted training sets, where the number $p$ of examples ('questions' with corresponding 'answers') scales linearly with the number $N$ of inputs, i.e. $p=\alpha N$. Here individual questions will re-appear during the learning process as soon as the number of weight updates made is of the order of the size of the training set. In the traditional models, where the duration of an update is defined as $N^{-1}$, this happens as soon as $t=\mathcal{O}(\alpha)$. At that point correlations develop between the weights and the questions in the training set, and the dynamics is of a spin-glass type, with the composition of the training set playing the role of 'quenched disorder'. The main consequence of this is that the central limit theorem no longer applies to the student's local fields, which are now described by non-Gaussian distributions. To demonstrate this we 
trained (on-line) a perceptron with weights $J_{i}$ on noiseless examples generated by a teacher perceptron with weights $B_{i}$, using the Hebb and AdaTron rules. We plotted in Fig. 1 the student and teacher fields, $x=\boldsymbol{J} \cdot \boldsymbol{\xi}$ and $y=\boldsymbol{B} \cdot \boldsymbol{\xi}$ respectively, where $\boldsymbol{\xi}$ is the input vector, for $p=N / 2$ examples and at time $t=50$. The marginal distribution $P(x)$ for $p=N / 4$, at times $t=10$ for the Hebb rule and $t=20$ for the Adatron rule, is shown in Fig. 2. The non-Gaussian student field distributions observed in Figs. 1 and 2 induce a deviation between the training- and generalization errors, which measure the network performance on training and test examples, respectively. The former involves averages over the non-Gaussian field distribution, whereas the latter (which is calculated over all possible examples) still involves Gaussian fields.

The appearance of non-Gaussian fields leads to a breakdown of the standard formalism, based on deriving closed equations for a finite number of observables: the field distributions can no longer be characterized by a few moments, and the macroscopic laws must now be averaged over realizations of the training set. One could still try to use Gaussian distributions as large $\alpha$ approximations, see e.g. Sollich and Barber (1998), but it will be clear from Figs. 1 and 2 that a systematic theory will have to give up Gaussian distributions entirely. The first rigorous study of the dynamics of learning with restricted training sets in non-linear networks, via the calculation of generating functionals, was carried out by Horner (1992) for perceptrons with binary weights. In this paper we show how the formalism of dynamical replica theory (see e.g. Coolen et al, 1996) can be used successfully to predict the evolution of macroscopic observables for finite $\alpha$, incorporating the infinite training set formalism as a special case, for $\alpha \rightarrow \infty$. Central to our approach is the derivation of a diffusion equation for the joint distribution of the student and teacher fields, which will be found to have Gaussian solutions only for $\alpha \rightarrow \infty$. For simplicity and transparency we restrict ourselves to single-layer systems and noise-free teachers. Application and generalization of our methods to multi-layer systems (Saad and Coolen, 1998) and learning scenarios involving 'noisy' teachers (Mace and Coolen, 1998b) are presently under way.

This paper is organized as follows. In section 2 we first derive a FokkerPlanck equation describing the evolution of arbitrary mean-field observables for $N \rightarrow \infty$. This allows us to identify the conditions for the latter to be described by closed deterministic laws. In section 3 we choose as our observables the joint field distribution $P[x, y]$, in addition to (the traditional ones) $Q$ and $R$, and show that this set obeys deterministic laws. In order to close these laws we use the tools of dynamical replica theory. Details of the replica calculation are given in section 4 , to be skipped by those primarily interested in results. In section 5 we show how in the limit $\alpha \rightarrow \infty$ (infinite training sets) the equations of the conventional theory are recovered. Finally we work out our equations explicitly for the example of on-line Hebbian learning with restricted training sets, and compare our predictions with exact results (derived directly from the microscopic equations) and with numerical simulations. 

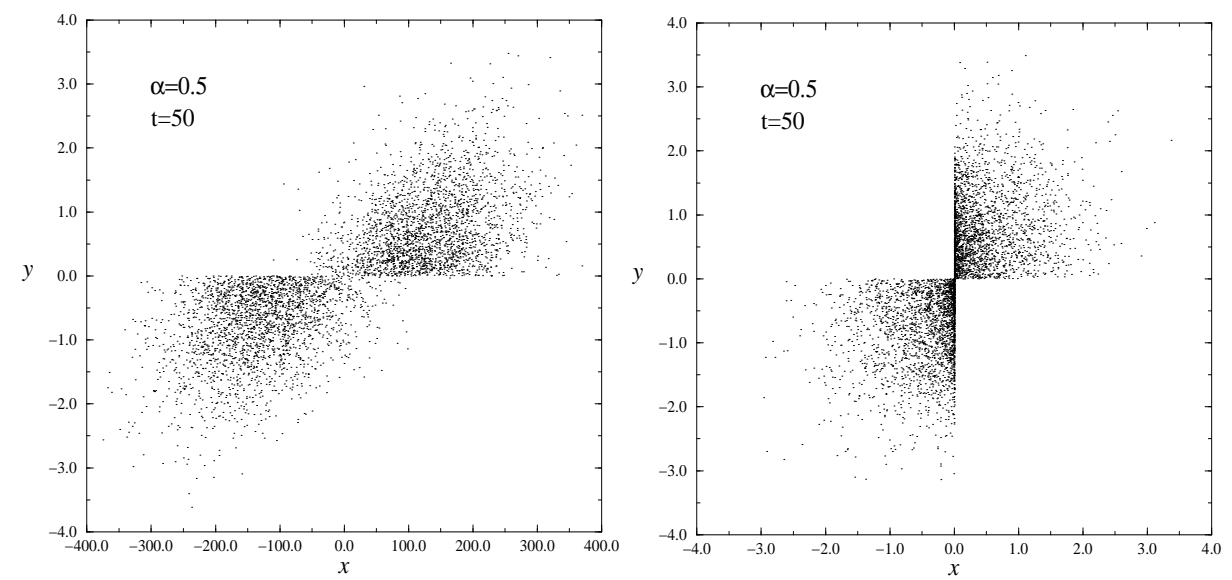

Figure 1: Fig. 1: Student and teacher fields $(x, y)$ as observed during numerical simulations of on-line learning (learning rate $\eta=1$ ) in a perceptron of size $N=10,000$ at $t=50$, using 'questions' from a restricted training set of size $p=\frac{1}{2} N$. Left: Hebbian learning. Right: AdaTron learning. Note: in the case of Gaussian field distributions one would have found spherically shaped plots.
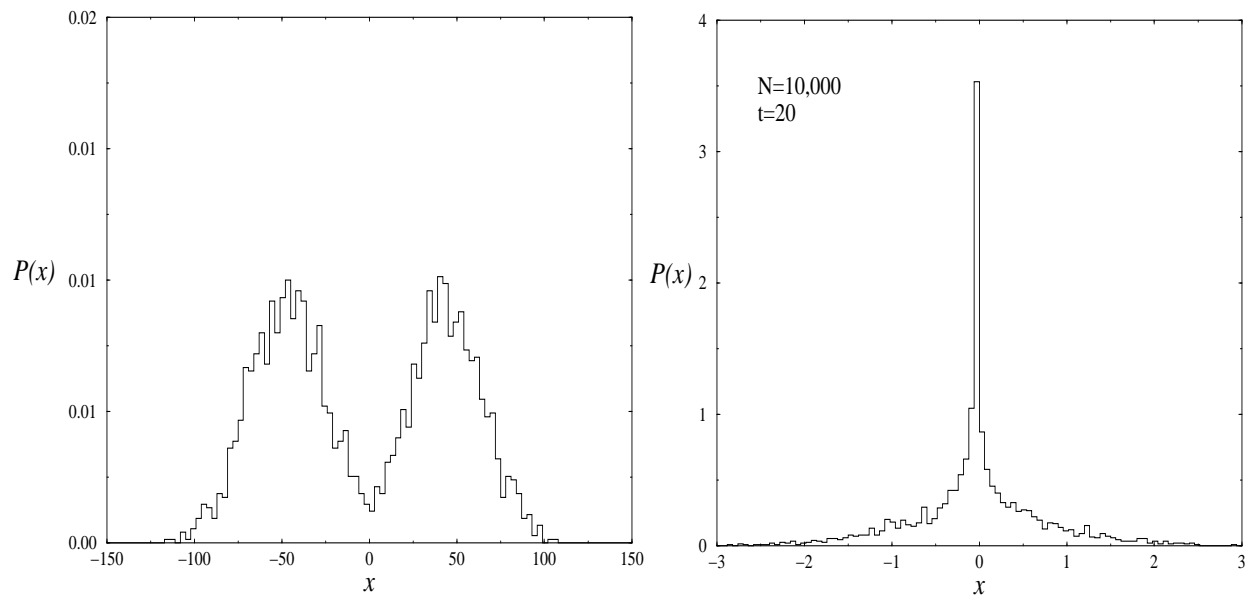

Figure 2: Fig. 2: Distribution $P(x)$ of student fields as observed during numerical simulations of on-line learning (learning rate $\eta=1$ ) in a perceptron of size $N=10,000$, using 'questions' from a restricted training set of size $p=\frac{1}{4} N$. Left: Hebbian learning, measured at $t=10$. Right: AdaTron learning, measured at $t=20$. Note: not only are these distributions distinctively non-Gaussian, they also appear to vary widely in their basic characteristics, depending on the learning rule used. 


\section{From Microscopic to Macroscopic Laws}

\subsection{Definitions}

A student perceptron operates the following rule, which is parametrised by the weight vector $\boldsymbol{J} \in \Re^{N}$ :

$$
S:\{-1,1\}^{N} \rightarrow\{-1,1\} \quad S(\boldsymbol{\xi})=\operatorname{sgn}[\boldsymbol{J} \cdot \boldsymbol{\xi}]
$$

It tries to emulate the operation of a teacher perceptron, via an iterative procedure for updating its parameters $\boldsymbol{J}$. The teacher perceptron operates a similar rule, characterized by a given (fixed) weight vector $\boldsymbol{B} \in \Re^{N}$ :

$$
T:\{-1,1\}^{N} \rightarrow\{-1,1\} \quad T(\boldsymbol{\xi})=\operatorname{sgn}[\boldsymbol{B} \cdot \boldsymbol{\xi}]
$$

In order to do so, the student perceptron modifies its weight vector $\boldsymbol{J}$ accord-

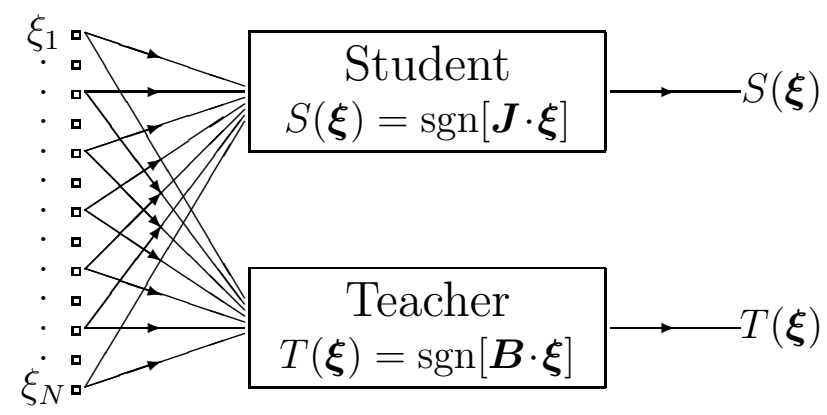

Figure 3: Fig. 3: Supervised learning in perceptrons.

ing to an iterative procedure, using examples of input vectors (or 'questions') $\boldsymbol{\xi}$, drawn at random from a fixed training set $\tilde{D} \subseteq D=\{-1,1\}^{N}$, and the corresponding values of the teacher outputs $T(\boldsymbol{\xi})$, see Fig. 3 .

We consider the case where the training set is a randomly composed subset $\tilde{D} \subset D$, of size $|\tilde{D}|=p=\alpha N$ with $\alpha>0$ :

$$
\tilde{D}=\left\{\boldsymbol{\xi}^{1}, \ldots, \boldsymbol{\xi}^{p}\right\} \quad p=\alpha N \quad \boldsymbol{\xi}^{\mu} \in D \text { for all } \mu
$$

We will denote averages over the training set $\tilde{D}$ and averages over the full question set $D$ in the following way:

$$
\langle\Phi(\boldsymbol{\xi})\rangle_{\tilde{D}}=\frac{1}{|\tilde{D}|} \sum_{\boldsymbol{\xi} \in \tilde{D}} \Phi(\boldsymbol{\xi}) \quad \text { and } \quad\langle\Phi(\boldsymbol{\xi})\rangle_{D}=\frac{1}{|D|} \sum_{\boldsymbol{\xi} \in D} \Phi(\boldsymbol{\xi}) .
$$

We will analyze the following two classes of learning rules:

$$
\begin{array}{lll}
\text { on-line : } & \boldsymbol{J}(m+1)=\boldsymbol{J}(m)+\frac{\eta}{N} \boldsymbol{\xi}(m) \mathcal{G}[\boldsymbol{J}(m) \cdot \boldsymbol{\xi}(m), \boldsymbol{B} \cdot \boldsymbol{\xi}(m)] \\
\text { batch : } & \boldsymbol{J}(m+1)=\boldsymbol{J}(m)+\frac{\eta}{N}\langle\boldsymbol{\xi} \mathcal{G}[\boldsymbol{J}(m) \cdot \boldsymbol{\xi}, \boldsymbol{B} \cdot \boldsymbol{\xi}]\rangle_{\tilde{D}}
\end{array}
$$


In on-line learning one draws at each iteration step $m$ a question $\boldsymbol{\xi}(m) \in \tilde{D}$ at random, the dynamics is thus a stochastic process; in batch learning one iterates a deterministic map. The function $\mathcal{G}[x, y]$ is assumed to be bounded and not to depend on $N$, other than via its two arguments.

Our most important observables during learning are the training error $E_{\mathrm{t}}(\boldsymbol{J})$ and the generalization error $E_{\mathrm{g}}(\boldsymbol{J})$, defined as follows:

$$
\begin{aligned}
& E_{\mathrm{t}}(\boldsymbol{J})=\langle\theta[-(\boldsymbol{J} \cdot \boldsymbol{\xi})(\boldsymbol{B} \cdot \boldsymbol{\xi})]\rangle_{\tilde{D}}, \\
& E_{\mathrm{g}}(\boldsymbol{J})=\langle\theta[-(\boldsymbol{J} \cdot \boldsymbol{\xi})(\boldsymbol{B} \cdot \boldsymbol{\xi})]\rangle_{D} .
\end{aligned}
$$

Only if the training set $\tilde{D}$ is sufficiently large, and if there are no correlations between $\boldsymbol{J}$ and the questions $\boldsymbol{\xi} \in \tilde{D}$, will these two errors will be identical.

\subsection{From Discrete to Continuous Time}

We next convert the dynamical laws (2.4) into the language of stochastic processes. We introduce the probability $\hat{p}_{m}(\boldsymbol{J})$ to find weight vector $\boldsymbol{J}$ at discrete iteration step $m$. In terms of this microscopic probability distribution the processes (2.4) can be written in the general Markovian form

$$
\hat{p}_{m+1}(\boldsymbol{J})=\int d \boldsymbol{J}^{\prime} W\left[\boldsymbol{J} ; \boldsymbol{J}^{\prime}\right] \hat{p}_{m}\left(\boldsymbol{J}^{\prime}\right),
$$

with the transition probabilities

$$
\begin{array}{ll}
\text { on-line : } & W\left[\boldsymbol{J} ; \boldsymbol{J}^{\prime}\right]=\left\langle\delta\left[\boldsymbol{J}-\boldsymbol{J}^{\prime}-\frac{\eta}{N} \boldsymbol{\xi} \mathcal{G}\left[\boldsymbol{J}^{\prime} \cdot \boldsymbol{\xi}, \boldsymbol{B} \cdot \boldsymbol{\xi}\right]\right]\right\rangle_{\tilde{D}} \\
\text { batch : } & W\left[\boldsymbol{J} ; \boldsymbol{J}^{\prime}\right]=\delta\left[\boldsymbol{J}-\boldsymbol{J}^{\prime}-\frac{\eta}{N}\left\langle\boldsymbol{\xi} \mathcal{G}\left[\boldsymbol{J}^{\prime} \cdot \boldsymbol{\xi}, \boldsymbol{B} \cdot \boldsymbol{\xi}\right]\right\rangle_{\tilde{D}}\right]
\end{array}
$$

We now make the transition to a description involving real-valued time labels by choosing the duration of each iteration step to be a real-valued random number, such that the probability that at time $t$ precisely $m$ steps have been made is given by the Poisson expression

$$
\pi_{m}(t)=\frac{1}{m !}(N t)^{m} e^{-N t} .
$$

For times $t \gg N^{-1}$ we find $t=m / N+\mathcal{O}\left(N^{-\frac{1}{2}}\right)$, the usual time unit. Due to the random durations of the iteration steps we have to switch to the following microscopic probability distribution:

$$
p_{t}(\boldsymbol{J})=\sum_{m \geq 0} \pi_{m}(t) \hat{p}_{m}(\boldsymbol{J}) .
$$

This distribution obeys a simple differential equation, which immediately follows from the pleasant properties of (2.9) under temporal differentiation:

$$
\frac{d}{d t} p_{t}(\boldsymbol{J})=N \int d \boldsymbol{J}^{\prime}\left\{W\left[\boldsymbol{J} ; \boldsymbol{J}^{\prime}\right]-\delta\left[\boldsymbol{J}-\boldsymbol{J}^{\prime}\right]\right\} p_{t}\left(\boldsymbol{J}^{\prime}\right) .
$$

So far no approximations have been made, equation (2.11) is exact for any $N$. It is the equivalent of the master equation often introduced to define the dynamics of spin systems. 


\subsection{Derivation of Macroscopic Fokker-Planck Equation}

We now wish to investigate the dynamics of a number of as yet arbitrary macroscopic observables $\boldsymbol{\Omega}[\boldsymbol{J}]=\left(\Omega_{1}[\boldsymbol{J}], \ldots, \Omega_{k}[\boldsymbol{J}]\right)$. To do so we introduce a macroscopic probability distribution

$$
P_{t}(\boldsymbol{\Omega})=\int d \boldsymbol{J} p_{t}(\boldsymbol{J}) \delta[\boldsymbol{\Omega}-\boldsymbol{\Omega}[\boldsymbol{J}]]
$$

Its time derivative immediately follows from that in (2.11):

$$
\frac{d}{d t} P_{t}(\boldsymbol{\Omega})=\int d \mathbf{\Omega}^{\prime} \mathcal{W}_{t}\left[\mathbf{\Omega} ; \mathbf{\Omega}^{\prime}\right] P_{t}\left(\mathbf{\Omega}^{\prime}\right)
$$

where

$\mathcal{W}_{t}\left[\boldsymbol{\Omega} ; \boldsymbol{\Omega}^{\prime}\right]=\frac{\int d \boldsymbol{J}^{\prime} p_{t}\left(\boldsymbol{J}^{\prime}\right) \delta\left[\boldsymbol{\Omega}^{\prime}-\boldsymbol{\Omega}\left[\boldsymbol{J}^{\prime}\right]\right] \int d \boldsymbol{J} \delta[\boldsymbol{\Omega}-\boldsymbol{\Omega}[\boldsymbol{J}]] N\left\{W\left[\boldsymbol{J} ; \boldsymbol{J}^{\prime}\right]-\delta\left[\boldsymbol{J}-\boldsymbol{J}^{\prime}\right]\right\}}{\int d \boldsymbol{J}^{\prime} p_{t}\left(\boldsymbol{J}^{\prime}\right) \delta\left[\boldsymbol{\Omega}^{\prime}-\boldsymbol{\Omega}\left[\boldsymbol{J}^{\prime}\right]\right]}$

If we insert the relevant expressions (2.8) for $W\left[\boldsymbol{J} ; \boldsymbol{J}^{\prime}\right]$ we can perform the $\boldsymbol{J}$ integrations, and obtain expressions in terms of so-called sub-shell averages, defined as

$$
\langle f(\boldsymbol{J})\rangle_{\boldsymbol{\Omega} ; t}=\frac{\int d \boldsymbol{J} p_{t}(\boldsymbol{J}) \delta[\boldsymbol{\Omega}-\boldsymbol{\Omega}[\boldsymbol{J}]] f(\boldsymbol{J})}{\int d \boldsymbol{J} p_{t}(\boldsymbol{J}) \delta[\boldsymbol{\Omega}-\boldsymbol{\Omega}[\boldsymbol{J}]]} .
$$

For the two types of learning rules at hand we obtain:

$$
\begin{aligned}
& \left.\mathcal{W}_{t}^{\text {onl }}\left[\boldsymbol{\Omega} ; \boldsymbol{\Omega}^{\prime}\right]=N\left\langle\delta\left[\boldsymbol{\Omega}-\boldsymbol{\Omega}\left[\boldsymbol{J}+\frac{\eta}{N} \boldsymbol{\xi} \mathcal{G}[\boldsymbol{J} \cdot \boldsymbol{\xi}, \boldsymbol{B} \cdot \boldsymbol{\xi}]\right]\right]\right\rangle_{\tilde{D}}-\delta[\boldsymbol{\Omega}-\boldsymbol{\Omega}[\boldsymbol{J}]]\right\rangle_{\boldsymbol{\Omega}^{\prime} ; t} \\
& \mathcal{W}_{t}^{\text {bat }}\left[\boldsymbol{\Omega} ; \boldsymbol{\Omega}^{\prime}\right]=N\left\langle\delta\left[\boldsymbol{\Omega}-\boldsymbol{\Omega}\left[\boldsymbol{J}+\frac{\eta}{N}\langle\boldsymbol{\xi} \mathcal{G}[\boldsymbol{J} \cdot \boldsymbol{\xi}, \boldsymbol{B} \cdot \boldsymbol{\xi}]\rangle_{\Omega}\right]\right]-\delta[\boldsymbol{\Omega}-\boldsymbol{\Omega}[\boldsymbol{J}]]\right\rangle_{\boldsymbol{\Omega}^{\prime} ; t}
\end{aligned}
$$

We now insert integral representations for the $\delta$-distributions. This gives for our two learning scenario's:

$$
\begin{gathered}
\left.\mathcal{W}_{t}^{\text {onl }}\left[\boldsymbol{\Omega} ; \boldsymbol{\Omega}^{\prime}\right]=\int \frac{d \hat{\boldsymbol{\Omega}}}{(2 \pi)^{k}} e^{i \hat{\boldsymbol{\Omega}} \cdot \boldsymbol{\Omega}} N\left\langle e^{-i \hat{\boldsymbol{\Omega}} \cdot \boldsymbol{\Omega}\left[\boldsymbol{J}+\frac{\eta}{N} \boldsymbol{\xi}[\boldsymbol{J} \cdot \boldsymbol{\xi}, \boldsymbol{B} \cdot \boldsymbol{\xi}]\right]}\right\rangle_{\tilde{D}}-e^{-i \hat{\boldsymbol{\Omega}} \cdot \boldsymbol{\Omega}[\boldsymbol{J}]}\right\rangle_{\boldsymbol{\Omega}^{\prime} ; t} \\
\mathcal{W}_{t}^{\text {bat }}\left[\boldsymbol{\Omega} ; \boldsymbol{\Omega}^{\prime}\right]=\int \frac{d \hat{\boldsymbol{\Omega}}}{(2 \pi)^{k}} e^{i \hat{\boldsymbol{\Omega}} \cdot \boldsymbol{\Omega}} N\left\langle e^{-i \hat{\boldsymbol{\Omega}} \cdot \boldsymbol{\Omega}\left[\boldsymbol{J}+\frac{\eta}{N}\langle\boldsymbol{\xi} \mathcal{G}[\boldsymbol{J} \cdot \boldsymbol{\xi}, \boldsymbol{B} \cdot \boldsymbol{\xi}]\rangle_{\tilde{D}}\right]}-e^{-i \hat{\boldsymbol{\Omega}} \cdot \boldsymbol{\Omega}[\boldsymbol{J}]}\right\rangle_{\boldsymbol{\Omega}^{\prime} ; t}
\end{gathered}
$$

Still no approximations have been made. The above two expressions differ only in the stage where the averaging over the training set is carried out.

In expanding equations $(2.14,2.15)$ for large $N$ and finite $t$ we have to be careful, since the system size $N$ enters both as a small parameter to control the magnitude of the modification of individual components of the weight 
vector, but also determines the dimensions and lengths of various vectors that occur. We therefore inspect more closely the usual Taylor expansions:

$$
F[\boldsymbol{J}+\boldsymbol{k}]=\sum_{\ell \geq 0} \frac{1}{\ell !} \sum_{i_{1}=1}^{N} \cdots \sum_{i_{\ell}=1}^{N} k_{i_{1}} \cdots k_{i_{\ell}} \frac{\partial^{\ell} F[\boldsymbol{J}]}{\partial J_{i_{1}} \cdots \partial J_{i_{\ell}}}
$$

If all derivatives were to be treated as $\mathcal{O}(1)$ (i.e. if we only take into account the scaling of the shift $\boldsymbol{k}$ with $N)$, problems could arise, since in the cases of interest (where $\boldsymbol{k}^{2}=\mathcal{O}\left(N^{-1}\right)$ ) this series could diverge as $F[\boldsymbol{J}+\boldsymbol{k}]=$ $\sum_{\ell \geq 0}\left(\sum_{i} k_{i}\right)^{\ell}=\sum_{\ell \geq 0} \mathcal{O}(1)$. If we assess how derivatives with respect to individual components $J_{i}$ scale for the standard types of mean-field observables, we find the following scaling property which we will choose as our definition of mean-field observables:

$$
\frac{\partial^{\ell} F[\boldsymbol{J}]}{\partial J_{i_{1}} \cdots \partial J_{i_{\ell}}}=\mathcal{O}\left(\frac{F[\boldsymbol{J}]}{|\boldsymbol{J}|^{\ell}} N^{\frac{1}{2} \ell-d}\right) \quad(N \rightarrow \infty)
$$

in which $d$ is the number of different elements in the set $\left\{i_{1}, \ldots, i_{\ell}\right\}$. If $F[\boldsymbol{J}]$ is a mean-field observable in the sense of (2.16), we can estimate the scaling of the various terms in the Taylor expansion:

$$
F[\boldsymbol{J}+\boldsymbol{k}]=F[\boldsymbol{J}]+\sum_{i} k_{i} \frac{\partial F[\boldsymbol{J}]}{\partial J_{i}}+\frac{1}{2} \sum_{i j} k_{i} k_{j} \frac{\partial^{2} F[\boldsymbol{J}]}{\partial J_{i} \partial J_{j}}+\sum_{\ell \geq 3} \mathcal{O}\left(F[\boldsymbol{J}]\left[\frac{|\boldsymbol{k}|}{|\boldsymbol{J}|}\right]^{\ell}\right)
$$

(in the last step we have used $\sum_{i} k_{i}=\mathcal{O}(\sqrt{N}|\boldsymbol{k}|)$ ).

We apply (2.17) to our macroscopic equations (2.14,2.15), restricting ourselves from now on to mean-field observables $\Omega[\boldsymbol{J}]=\mathcal{O}\left(N^{0}\right)$ in the sense of (2.16), one of which we choose to be $\boldsymbol{J}^{2}$. Here the shifts $\boldsymbol{k}$, being either $\frac{\eta}{N} \boldsymbol{\xi} \mathcal{G}[\boldsymbol{J} \cdot \boldsymbol{\xi} ; \boldsymbol{B} \cdot \boldsymbol{\xi}]$ or $\frac{\eta}{N}\langle\boldsymbol{\xi} \mathcal{G}[\boldsymbol{J} \cdot \boldsymbol{\xi} ; \boldsymbol{B} \cdot \boldsymbol{\xi}]\rangle_{\tilde{D}}$, scale as $|\boldsymbol{k}|=\mathcal{O}\left(N^{-\frac{1}{2}}\right)$. Consequently the $\ell$-th order term in the expansions of both (2.14) and (2.15) will be of order $N^{-\frac{\ell}{2}}$ :

$$
\begin{aligned}
& e^{-i \hat{\boldsymbol{\Omega}} \cdot \boldsymbol{\Omega}[\boldsymbol{J}+\boldsymbol{k}]}=e^{-i \hat{\boldsymbol{\Omega}} \cdot \boldsymbol{\Omega}[\boldsymbol{J}]}\left\{1-i \sum_{i} k_{i} \frac{\partial}{\partial J_{i}}(\hat{\boldsymbol{\Omega}} \cdot \boldsymbol{\Omega}[\boldsymbol{J}])\right. \\
& \left.\quad-\frac{i}{2} \sum_{i j} k_{i} k_{j} \frac{\partial^{2}}{\partial J_{i} \partial J_{j}}(\hat{\boldsymbol{\Omega}} \cdot \boldsymbol{\Omega}[\boldsymbol{J}])-\frac{1}{2}\left[\sum_{i} k_{i} \frac{\partial}{\partial J_{i}}(\hat{\boldsymbol{\Omega}} \cdot \boldsymbol{\Omega}[\boldsymbol{J}])\right]^{2}\right\}+\mathcal{O}\left(N^{-\frac{3}{2}}\right) .
\end{aligned}
$$

This, in turn, gives

$$
\begin{aligned}
& \int \frac{d \hat{\boldsymbol{\Omega}}}{(2 \pi)^{k}} e^{i \hat{\boldsymbol{\Omega}} \cdot \boldsymbol{\Omega}} N\left[e^{-i \hat{\boldsymbol{\Omega}} \cdot \boldsymbol{\Omega}\left[\boldsymbol{J}_{+} \boldsymbol{k}\right]}-e^{-i \hat{\boldsymbol{\Omega}} \cdot \boldsymbol{\Omega}[\boldsymbol{J}]}\right] \\
& \quad=-N\left\{\sum_{\mu} \frac{\partial}{\partial \Omega_{\mu}}\left[\sum_{i} k_{i} \frac{\partial \Omega_{\mu}[\boldsymbol{J}]}{\partial J_{i}}+\frac{1}{2} \sum_{i j} k_{i} k_{j} \frac{\partial^{2} \Omega_{\mu}[\boldsymbol{J}]}{\partial J_{i} \partial J_{j}}\right]\right.
\end{aligned}
$$




$$
\left.-\frac{1}{2} \sum_{\mu \nu} \frac{\partial^{2}}{\partial \Omega_{\mu} \partial \Omega_{\nu}} \sum_{i j} k_{i} k_{j} \frac{\partial \Omega_{\mu}[\boldsymbol{J}]}{\partial J_{i}} \frac{\partial \Omega_{\nu}[\boldsymbol{J}]}{\partial J_{j}}\right\} \delta[\boldsymbol{\Omega}-\boldsymbol{\Omega}[\boldsymbol{J}]]+\mathcal{O}\left(N^{-\frac{1}{2}}\right) .
$$

It is now evident, in view of (2.14,2.15), that both types of dynamics are described by macroscopic laws with transition probability densities of the general form

$$
\begin{aligned}
\mathcal{W}_{t}^{\star \star \star}\left[\Omega ; \Omega^{\prime}\right]=\left\{-\sum_{\mu} F_{\mu}\left[\Omega^{\prime} ; t\right] \frac{\partial}{\partial \Omega_{\mu}}+\frac{1}{2} \sum_{\mu \nu} G_{\mu \nu}\left[\Omega^{\prime} ; t\right] \frac{\partial^{2}}{\partial \Omega_{\mu} \partial \Omega_{\nu}}\right\} & \delta\left[\Omega-\Omega^{\prime}\right] \\
& +\mathcal{O}\left(N^{-\frac{1}{2}}\right)
\end{aligned}
$$

which, due to (2.13) and for $N \rightarrow \infty$ and finite times, leads to a Fokker-Planck equation:

$$
\frac{d}{d t} P_{t}(\boldsymbol{\Omega})=-\sum_{\mu=1}^{k} \frac{\partial}{\partial \Omega_{\mu}}\left\{F_{\mu}[\boldsymbol{\Omega} ; t] P_{t}(\boldsymbol{\Omega})\right\}+\frac{1}{2} \sum_{\mu \nu=1}^{k} \frac{\partial^{2}}{\partial \Omega_{\mu} \partial \Omega_{\nu}}\left\{G_{\mu \nu}[\boldsymbol{\Omega} ; t] P_{t}(\boldsymbol{\Omega})\right\}
$$

The differences between the two types of dynamics are in the explicit expressions for the flow- and diffusion terms:

$$
\begin{aligned}
F_{\mu}^{\mathrm{onl}}[\boldsymbol{\Omega} ; t]= & \eta\left\langle\left\langle\sum_{i} \xi_{i} \mathcal{G}[\boldsymbol{J} \cdot \boldsymbol{\xi}, \boldsymbol{B} \cdot \boldsymbol{\xi}] \frac{\partial \Omega_{\mu}[\boldsymbol{J}]}{\partial J_{i}}\right\rangle_{\tilde{D}}\right\rangle_{\boldsymbol{\Omega} ; t} \\
& +\frac{\eta^{2}}{2 N}\left\langle\left\langle\sum_{i j} \xi_{i} \xi_{j} \mathcal{G}^{2}[\boldsymbol{J} \cdot \boldsymbol{\xi}, \boldsymbol{B} \cdot \boldsymbol{\xi}] \frac{\partial^{2} \Omega_{\mu}[\boldsymbol{J}]}{\partial J_{i} \partial J_{j}}\right\rangle_{\tilde{D}}\right\rangle_{\boldsymbol{\Omega} ; t} \\
G_{\mu \nu}^{\mathrm{onl}}[\boldsymbol{\Omega} ; t]= & \frac{\eta^{2}}{N}\left\langle\left\langle\sum_{i j} \xi_{i} \xi_{j} \mathcal{G}^{2}[\boldsymbol{J} \cdot \boldsymbol{\xi}, \boldsymbol{B} \cdot \boldsymbol{\xi}]\left[\frac{\partial \Omega_{\mu}[\boldsymbol{J}]}{\partial J_{i}}\right]\left[\frac{\partial \Omega_{\nu}[\boldsymbol{J}]}{\partial J_{j}}\right]\right\rangle_{\tilde{D}}\right\rangle_{\boldsymbol{\Omega} ; t} \\
F_{\mu}^{\mathrm{bat}}[\boldsymbol{\Omega} ; t]= & \eta\left\langle\sum_{i}\left\langle\xi_{i} \mathcal{G}[\boldsymbol{J} \cdot \boldsymbol{\xi}, \boldsymbol{B} \cdot \boldsymbol{\xi}]\right\rangle_{\tilde{D}} \frac{\partial \Omega_{\mu}[\boldsymbol{J}]}{\partial J_{i}}\right\rangle_{\boldsymbol{\Omega} ; t} \\
& +\frac{\eta^{2}}{2 N}\left\langle\sum_{i j}\left\langle\xi_{i} \mathcal{G}[\boldsymbol{J} \cdot \boldsymbol{\xi}, \boldsymbol{B} \cdot \boldsymbol{\xi}]\right\rangle_{\tilde{D}}\left\langle\xi_{j} \mathcal{G}[\boldsymbol{J} \cdot \boldsymbol{\xi}, \boldsymbol{B} \cdot \boldsymbol{\xi}]\right\rangle_{\tilde{D}} \frac{\partial^{2} \Omega_{\mu}[\boldsymbol{J}]}{\partial J_{i} \partial J_{j}}\right\rangle_{\boldsymbol{\Omega} ; t} \\
G_{\mu \nu}^{\mathrm{bat}}[\boldsymbol{\Omega} ; t]= & \frac{\eta^{2}}{N}\left\langle\sum_{i j}\left\langle\xi_{i} \mathcal{G}[\boldsymbol{J} \cdot \boldsymbol{\xi}, \boldsymbol{B} \cdot \boldsymbol{\xi}]\right\rangle_{\tilde{D}}\left\langle\xi_{j} \mathcal{G}[\boldsymbol{J} \cdot \boldsymbol{\xi}, \boldsymbol{B} \cdot \boldsymbol{\xi}]\right\rangle_{\tilde{D}}\left[\frac{\partial \Omega_{\mu}[\boldsymbol{J}]}{\partial J_{i}}\right]\left[\frac{\left.\partial \Omega_{\nu}[\boldsymbol{J}]\right]}{\partial J_{j}}\right]\right\rangle_{\boldsymbol{\Omega} ; t}
\end{aligned}
$$

Equation (2.18) allows us to define the goal of our exercise in more explicit form. If we wish to arrive at closed deterministic macroscopic equations, we have to choose our observables such that

$$
\begin{array}{ll}
\text { 1. } \lim _{N \rightarrow \infty} G_{\mu \nu}[\Omega ; t]=0 & \text { (this ensures determinism) } \\
\text { 2. } \lim _{N \rightarrow \infty} \frac{\partial}{\partial t} F_{\mu}[\Omega ; t]=0 & \text { (this ensures closure) }
\end{array}
$$

In the case of time-dependent global parameters, such as learning rates or decay rates, the latter condition relaxes to the requirement that any explicit time-dependence of $F_{\mu}[\Omega ; t]$ is restricted to these global parameters. 


\section{Application to Canonical Observables}

\subsection{Choice of Canonical Observables}

We now apply the general results obtained so far to a specific set of observables $\boldsymbol{\Omega}[\boldsymbol{J}]$, taylored to the problem at hand:

$$
Q[\boldsymbol{J}]=\boldsymbol{J}^{2}, \quad R[\boldsymbol{J}]=\boldsymbol{J} \cdot \boldsymbol{B}, \quad P[x, y ; \boldsymbol{J}]=\langle\delta[x-\boldsymbol{J} \cdot \boldsymbol{\xi}] \delta[x-\boldsymbol{B} \cdot \boldsymbol{\xi}]\rangle_{\tilde{D}}
$$

This choice is motivated by the following considerations: (i) in order to incorporate the standard theory in the limit $\alpha \rightarrow \infty$ we need at least $Q[\boldsymbol{J}]$ and $R[\boldsymbol{J}]$, (ii) we need to be able to calculate the training error, which involves field statistics calculated over the training set $\tilde{D}$, as described by $P[x, y ; \boldsymbol{J}]$, and (iii) for finite $\alpha$ one cannot expect closed macroscopic equations for just a finite number of order parameters, the present choice (involving the order parameter function $P[x, y ; \boldsymbol{J}]$ ) represents effectively an infinite numberf. In subsequent calculations we will, however, assume the number of arguments $(x, y)$ for which $P[x, y ; \boldsymbol{J}]$ is to be evaluated (and thus our number of order parameters) to go to infinity only after the limit $N \rightarrow \infty$ has been taken. This will eliminate many technical subtleties and will allow us to use the Fokker-Planck equation (2.18).

The observables (3.1) are indeed of the mean-field type in the sense of (2.16). Insertion into (2.16) immediately shows this to be true for the scalar observables $Q[\boldsymbol{J}]$ and $R[\boldsymbol{J}]$. Checking (2.16) for the function $P[x, y ; \boldsymbol{J}]$ is less trivial. Here we have to use the property that $\tilde{D}$ has been composed in a random manner. We denote with $\mathcal{I}$ the set of all different indices in the list $\left(i_{1}, \ldots, i_{\ell}\right)$, with $n_{k}$ giving the number of times a number $k$ occurs, and with $\mathcal{I}^{ \pm} \subseteq \mathcal{I}$ defined as the set of all indices $k \in \mathcal{I}$ for which $n_{k}$ is even $(+)$, or odd $(-)$. Note that with these definitions $\ell=\sum_{k \in \mathcal{I}^{+}} n_{k}+\sum_{k \in \mathcal{I}^{-}} n_{k} \geq 2\left|\mathcal{I}^{+}\right|+\left|\mathcal{I}^{-}\right|$. We then obtain the following scaling identity:

$$
\begin{aligned}
\left\langle\xi_{i_{1}} \ldots \xi_{i_{\ell}} \delta[x-\boldsymbol{J} \cdot \boldsymbol{\xi}] \delta[y-\boldsymbol{B} \cdot \boldsymbol{\xi}]\right\rangle_{\tilde{D}} & \\
= & i^{\ell} \int \frac{d \hat{x} d \hat{y}}{(2 \pi)^{2}} e^{i[x \hat{x}+y \hat{y}]}\left\langle\left[\prod_{k \in \mathcal{I}} \xi_{k}^{n_{k}} e^{-i \xi_{k}\left[\hat{x} J_{k}+\hat{y} B_{k}\right]}\right]\left[\prod_{k \notin \mathcal{I}} e^{-i \xi_{k}\left[\hat{x} J_{k}+\hat{y} B_{k}\right]}\right]\right\rangle_{\tilde{D}} \\
\quad= & i^{\ell} \int \frac{d \hat{x} d \hat{y}}{(2 \pi)^{2}} e^{i[x \hat{x}+y \hat{y}]} \mathcal{O}\left(N^{-\frac{1}{2}\left|\mathcal{I}^{-}\right|}\right)=\mathcal{O}\left(N^{-\frac{1}{2}\left|\mathcal{I}^{-}\right|}\right)
\end{aligned}
$$

This gives for derivatives of $P[x, y ; \boldsymbol{J}]$ :

$\frac{\partial^{\ell}}{\partial J_{i_{1}} \ldots \partial J_{i_{\ell}}} P[x, y ; \boldsymbol{J}]=(-1)^{\ell} \frac{\partial^{\ell}}{\partial x^{\ell}}\left\langle\xi_{i_{1}} \ldots \xi_{i_{\ell}} \delta[x-\boldsymbol{J} \cdot \boldsymbol{\xi}] \delta[y-\boldsymbol{B} \cdot \boldsymbol{\xi}]\right\rangle_{\tilde{D}}=\mathcal{O}\left(N^{-\frac{1}{2}\left|\mathcal{I}^{-}\right|}\right)$

Since $\frac{1}{2} \ell-|\mathcal{I}|+\frac{1}{2}\left|\mathcal{I}^{-}\right|=\frac{1}{2}\left[\ell-\left|\mathcal{I}^{-}\right|-2\left|\mathcal{I}^{+}\right|\right] \geq 0$, the function $P[x, y ; \boldsymbol{J}]$ is indeed found to be a mean-field observable.

\footnotetext{
${ }^{1} \mathrm{~A}$ simple rule of thumb is the following: if a process requires replica theory for its stationary state analysis, as does learning with restricted training sets, its dynamics is of a spin-glass type and cannot be described by a finite set of closed dynamic equations.
} 


\subsection{Deterministic Dynamical Laws}

We next show that for the observables (3.1) the diffusion matrix elements $G_{\mu \nu}^{\star \star \star}$ in the Fokker-Planck equation (2.18) vanish for $N \rightarrow \infty$. Our observables will consequently obey deterministic dynamical laws. The diffusion terms associated with $Q[\boldsymbol{J}]$ and $R[\boldsymbol{J}]$ are trivial. For on-line learning we find:

$$
\begin{aligned}
{\left[\begin{array}{l}
G_{Q Q}^{\mathrm{onl}}[\ldots] \\
G_{Q R}^{\mathrm{onl}}[\ldots] \\
G_{R R}^{\mathrm{onl}}[\ldots]
\end{array}\right] } & =\frac{\eta^{2}}{N}\left\langle\left\langle\sum_{i j} \xi_{i} \xi_{j} \mathcal{G}^{2}[\boldsymbol{J} \cdot \boldsymbol{\xi}, \boldsymbol{B} \cdot \boldsymbol{\xi}]\left[\begin{array}{c}
\left(2 J_{i}\right)\left(2 J_{j}\right) \\
\left(2 J_{i}\right)\left(B_{j}\right) \\
\left(B_{i}\right)\left(B_{j}\right)
\end{array}\right]\right\rangle_{\tilde{D}}\right\rangle_{\mathrm{QRP} ; t} \\
& =\frac{\eta^{2}}{N} \int d x d y P[x, y] \mathcal{G}^{2}[x, y]\left[\begin{array}{c}
4 x^{2} \\
2 x y \\
y^{2}
\end{array}\right]=\mathcal{O}\left(\frac{1}{N}\right)
\end{aligned}
$$

where the notation $\langle\cdots\rangle_{\mathrm{QRP} ; t}$ refers to sub-shell averages with respect to the order parameters $Q, R$ and $\{P[x, y]\}$ at time $t$. For batch learning, similarly:

$$
\begin{gathered}
{\left[\begin{array}{c}
G_{Q Q}^{\mathrm{bat}}[\ldots] \\
G_{Q R}^{\mathrm{bat}}[\ldots] \\
G_{R R}^{\mathrm{bat}}[\ldots]
\end{array}\right]=\frac{\eta^{2}}{N}\left\langle\sum_{i j}\left\langle\xi_{i} \mathcal{G}[\boldsymbol{J} \cdot \boldsymbol{\xi}, \boldsymbol{B} \cdot \boldsymbol{\xi}]\right\rangle_{\tilde{D}}\left\langle\xi_{j} \mathcal{G}[\boldsymbol{J} \cdot \boldsymbol{\xi}, \boldsymbol{B} \cdot \boldsymbol{\xi}]\right\rangle_{\tilde{D}}\left[\begin{array}{c}
\left(2 J_{i}\right)\left(2 J_{j}\right) \\
\left(2 J_{i}\right)\left(B_{j}\right) \\
\left(B_{i}\right)\left(B_{j}\right)
\end{array}\right]\right\rangle_{\mathrm{QRP} ; t}} \\
=\frac{\eta^{2}}{N}\left[\begin{array}{c}
4\left\{\int d x d y P[x, y] x \mathcal{G}[x, y]\right\}^{2} \\
\left.\left\{\int d x d y P[x ; y] x \mathcal{G}[x, y]\right\}\left\{\int d x d y P[x ; y] y \mathcal{G}[x, y]\right\}\right]=\mathcal{O}\left(\frac{1}{N}\right) \\
\left\{\int d x d y P[x ; y] y \mathcal{G}[x, y]\right\}^{2}
\end{array}\right]
\end{gathered}
$$

In calculating diffusion terms which involve the order parameter function $P[x ; y ; \boldsymbol{J}]$ we will again need the scaling property (3.2). First we turn to online learning diffusion terms with just one occurrence of $P[x, y ; \boldsymbol{J}]$

$$
\begin{gathered}
{\left[\begin{array}{c}
G_{Q, P[x, y]}^{\mathrm{onl}}[\cdots] \\
G_{R, P[x, y]}^{\mathrm{onl}}[\cdots]
\end{array}\right]=} \\
-\frac{\eta^{2}}{N}\left\langle\left\langle\sum_{i j} \xi_{i} \xi_{j} \mathcal{G}^{2}[\boldsymbol{J} \cdot \boldsymbol{\xi}, \boldsymbol{B} \cdot \boldsymbol{\xi}] \frac{\partial}{\partial x}\left[\begin{array}{c}
2 J_{i}\left\langle\xi_{j}^{\prime} \delta\left[x-\boldsymbol{J} \cdot \boldsymbol{\xi}^{\prime}\right] \delta\left[y-\boldsymbol{B} \cdot \boldsymbol{\xi}^{\prime}\right]\right\rangle_{\tilde{D}} \\
B_{i}\left\langle\xi_{j}^{\prime} \delta\left[x-\boldsymbol{J} \cdot \boldsymbol{\xi}^{\prime}\right] \delta\left[y-\boldsymbol{B} \cdot \boldsymbol{\xi}^{\prime}\right]\right\rangle_{\tilde{D}}
\end{array}\right]\right\rangle_{\tilde{D}}\right\rangle_{\mathrm{QRP} ; t} \\
=-\eta^{2} \int d x d y P[x, y] \mathcal{G}^{2}[x, y]\left[\begin{array}{c}
2 x \\
y
\end{array}\right] \mathcal{O}\left(\frac{1}{\sqrt{N}}\right)=\mathcal{O}\left(\frac{1}{\sqrt{N}}\right)
\end{gathered}
$$

For batch learning we find a similar result:

$$
\begin{aligned}
& {\left[\begin{array}{l}
G_{Q, P[x, y]}^{\mathrm{bat}}[\cdots] \\
G_{R, P[x, y]}^{\mathrm{bat}}[\cdots]
\end{array}\right]=-\frac{\eta^{2}}{N}\left\langle\sum_{i j}\left\langle\xi_{i} \mathcal{G}[\boldsymbol{J} \cdot \boldsymbol{\xi}, \boldsymbol{B} \cdot \boldsymbol{\xi}]\right\rangle_{\tilde{D}}\left\langle\xi_{j} \mathcal{G}[\boldsymbol{J} \cdot \boldsymbol{\xi}, \boldsymbol{B} \cdot \boldsymbol{\xi}]\right\rangle_{\tilde{D}}\right.}
\end{aligned}
$$

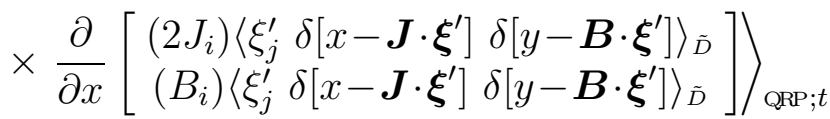

$$
\begin{aligned}
& =-\eta^{2} \int d x d y P[x, y] \mathcal{G}[x, y]\left[\begin{array}{c}
2 x \\
y
\end{array}\right] \mathcal{O}\left(\frac{1}{\sqrt{N}}\right)=\mathcal{O}\left(\frac{1}{\sqrt{N}}\right)
\end{aligned}
$$


The non-trivial terms are those where two derivatives of the order parameter function $P[x, y ; \boldsymbol{J}]$ come into play. These are dealt with by separating $i=j$ from $i \neq j$ terms, in combination with (3.2):

$$
\begin{aligned}
G_{P[x, y], P\left[x^{\prime}, y^{\prime}\right]}^{\mathrm{onl}}[\ldots] & =\frac{\eta^{2}}{N}\left\langle\left\langle\sum_{i j} \xi_{i} \xi_{j} \mathcal{G}^{2}[\boldsymbol{J} \cdot \boldsymbol{\xi}, \boldsymbol{B} \cdot \boldsymbol{\xi}]\right\rangle_{\tilde{D}} \frac{\partial^{2}}{\partial x \partial x^{\prime}}\right. \\
& \left.\left\langle\xi_{i}^{\prime} \delta\left[x-\boldsymbol{J} \cdot \boldsymbol{\xi}^{\prime}\right] \delta\left[y-\boldsymbol{B} \cdot \boldsymbol{\xi}^{\prime}\right]\right\rangle_{\tilde{D}}\left\langle\xi_{j}^{\prime} \delta\left[x^{\prime}-\boldsymbol{J} \cdot \boldsymbol{\xi}^{\prime}\right] \delta\left[y^{\prime}-\boldsymbol{B} \cdot \boldsymbol{\xi}^{\prime}\right]\right\rangle_{\tilde{D}}\right\rangle_{\mathrm{QRP} ; t} \\
& =\frac{\eta^{2}}{N} \frac{\partial^{2}}{\partial x \partial x^{\prime}}\left\langle\sum_{i} \mathcal{O}\left(N^{-1}\right)+\sum_{i \neq j} \mathcal{O}\left(N^{-2}\right)\right\rangle_{\mathrm{QRP} ; t}=\mathcal{O}\left(N^{-1}\right)
\end{aligned}
$$

Similarly:

$$
\begin{aligned}
G_{P[x, y], P\left[x^{\prime}, y^{\prime}\right]}^{\mathrm{bat}}[\ldots] & =\frac{\eta^{2}}{N}\left\langle\sum_{i j}\left\langle\xi_{i} \mathcal{G}[\boldsymbol{J} \cdot \boldsymbol{\xi}, \boldsymbol{B} \cdot \boldsymbol{\xi}]\right\rangle_{\tilde{D}}\left\langle\xi_{j} \mathcal{G}[\boldsymbol{J} \cdot \boldsymbol{\xi}, \boldsymbol{B} \cdot \boldsymbol{\xi}]\right\rangle_{\tilde{D}} \frac{\partial^{2}}{\partial x \partial x^{\prime}}\right. \\
& \left.\left\langle\xi_{i}^{\prime} \delta\left[x-\boldsymbol{J} \cdot \boldsymbol{\xi}^{\prime}\right] \delta\left[y-\boldsymbol{B} \cdot \boldsymbol{\xi}^{\prime}\right]\right\rangle_{\tilde{D}}\left\langle\xi_{j}^{\prime} \delta\left[x^{\prime}-\boldsymbol{J} \cdot \boldsymbol{\xi}^{\prime}\right] \delta\left[y^{\prime}-\boldsymbol{B} \cdot \boldsymbol{\xi}^{\prime}\right]\right\rangle_{\tilde{D}}\right\rangle_{\mathrm{QRP} ; t} \\
& =\frac{\eta^{2}}{N} \frac{\partial^{2}}{\partial x \partial x^{\prime}}\left\langle\sum_{i} \mathcal{O}\left(N^{-2}\right)+\sum_{i \neq j} \mathcal{O}\left(N^{-2}\right)\right\rangle_{\mathrm{QRP} ; t}=\mathcal{O}\left(N^{-1}\right)
\end{aligned}
$$

All diffusion terms vanish in the limit $N \rightarrow \infty$. The Fokker-Planck equation (2.18) reduces to the Liouville equation $\frac{d}{d t} P_{t}(\boldsymbol{\Omega})=-\sum_{\mu} \frac{\partial}{\partial \Omega_{\mu}}\left[F_{\mu}[\Omega ; t] P_{t}(\boldsymbol{\Omega})\right]$, describing deterministic evolution for our macroscopic observables: $\frac{d}{d t} \boldsymbol{\Omega}=$ $\boldsymbol{F}[\Omega ; t]$. These deterministic equations we will now work out explicitly.

\section{On-Line Learning}

First we deal with the scalar observables $Q$ and $R$ :

$$
\begin{aligned}
\frac{d}{d t} Q & =\lim _{N \rightarrow \infty}\left\{2 \eta\left\langle\langle(\boldsymbol{J} \cdot \boldsymbol{\xi}) \mathcal{G}[\boldsymbol{J} \cdot \boldsymbol{\xi}, \boldsymbol{B} \cdot \boldsymbol{\xi}]\rangle_{\tilde{D}}\right\rangle_{\mathrm{QRP} ; t}+\eta^{2}\left\langle\left\langle\mathcal{G}^{2}[\boldsymbol{J} \cdot \boldsymbol{\xi}, \boldsymbol{B} \cdot \boldsymbol{\xi}]\right\rangle_{\tilde{D}}\right\rangle_{\mathrm{QRP} ; t}\right\} \\
& =2 \eta \int d x d y P[x, y] x \mathcal{G}[x, y]+\eta^{2} \int d x d y P[x, y] \mathcal{G}^{2}[x, y] \\
\frac{d}{d t} R & =\lim _{N \rightarrow \infty} \eta\left\langle\langle(\boldsymbol{B} \cdot \boldsymbol{\xi}) \mathcal{G}[\boldsymbol{J} \cdot \boldsymbol{\xi}, \boldsymbol{B} \cdot \boldsymbol{\xi}]\rangle_{\tilde{D}}\right\rangle_{\mathrm{QRP} ; t}=\eta \int d x d y P[x, y] y \mathcal{G}[x, y]
\end{aligned}
$$

The equations (3.3,3.4) are identical to those found in the $\alpha \rightarrow \infty$ formalism. The difference is in the function to be substituted for $P[x, y]$, which here is the solution of

$$
\begin{gathered}
\frac{\partial}{\partial t} P[x, y]=\lim _{N \rightarrow \infty}\left\{-\eta \frac{\partial}{\partial x} \sum_{i}\left\langle\left\langle\xi_{i} \mathcal{G}[\boldsymbol{J} \cdot \boldsymbol{\xi}, \boldsymbol{B} \cdot \boldsymbol{\xi}]\right\rangle_{\tilde{D}}\left\langle\xi_{i}^{\prime} \delta\left[x-\boldsymbol{J} \cdot \boldsymbol{\xi}^{\prime}\right] \delta\left[y-\boldsymbol{B} \cdot \boldsymbol{\xi}^{\prime}\right]\right\rangle_{\tilde{D}}\right\rangle_{\mathrm{QRP} ; t}\right. \\
\left.+\frac{\eta^{2}}{2 N} \frac{\partial^{2}}{\partial x^{2}}\left\langle\sum_{i j}\left\langle\xi_{i} \xi_{j} \mathcal{G}^{2}[\boldsymbol{J} \cdot \boldsymbol{\xi}, \boldsymbol{B} \cdot \boldsymbol{\xi}]\right\rangle_{\tilde{D}}\left\langle\xi_{i}^{\prime} \xi_{j}^{\prime} \delta\left[x-\boldsymbol{J} \cdot \boldsymbol{\xi}^{\prime}\right] \delta\left[y-\boldsymbol{B} \cdot \boldsymbol{\xi}^{\prime}\right]\right\rangle_{\tilde{D}}\right\rangle_{\mathrm{QRP} ; t}\right\}
\end{gathered}
$$


According to $(3.2)$ the off-diagonal terms $i \neq j$ in the contribution with the second derivative $\frac{\partial^{2}}{\partial x^{2}}$ together contribute only vanishing orders $\left(N^{-1}\right)$, so that we need only consider the diagonal $i=j$ ones:

$$
\begin{gathered}
\frac{\partial}{\partial t} P[x, y]=\lim _{N \rightarrow \infty}\left\{-\eta \frac{\partial}{\partial x}\left\langle\left\langle\left\langle\mathcal{G}[\boldsymbol{J} \cdot \boldsymbol{\xi}, \boldsymbol{B} \cdot \boldsymbol{\xi}]\left(\boldsymbol{\xi} \cdot \boldsymbol{\xi}^{\prime}\right) \delta\left[x-\boldsymbol{J} \cdot \boldsymbol{\xi}^{\prime}\right] \delta\left[y-\boldsymbol{B} \cdot \boldsymbol{\xi}^{\prime}\right]\right\rangle_{\tilde{D}}\right\rangle_{\tilde{D}}\right\rangle_{\mathrm{QRP} ; t}\right. \\
\left.+\frac{1}{2} \eta^{2} \frac{\partial^{2}}{\partial x^{2}}\left\langle P[x, y ; \boldsymbol{J}]\left\langle\mathcal{G}^{2}[\boldsymbol{J} \cdot \boldsymbol{\xi}, \boldsymbol{B} \cdot \boldsymbol{\xi}]\right\rangle_{\tilde{D}}\right\rangle_{\mathrm{QRP} ; t}\right\} \\
=-\eta \frac{\partial}{\partial x} \int d x^{\prime} d y^{\prime} \mathcal{G}\left[x^{\prime}, y^{\prime}\right] \mathcal{C}\left[x, y ; x^{\prime}, y^{\prime}\right]+\frac{1}{2} \eta^{2} \int d x^{\prime} d y^{\prime} P\left[x^{\prime}, y^{\prime}\right] \mathcal{G}^{2}\left[x^{\prime}, y^{\prime}\right] \frac{\partial^{2}}{\partial x^{2}} P[x, y]
\end{gathered}
$$

with the function

$\mathcal{C}\left[x, y ; x^{\prime}, y^{\prime}\right]=\lim _{N \rightarrow \infty}\left\langle\left\langle\left\langle\delta[x-\boldsymbol{J} \cdot \boldsymbol{\xi}] \delta[y-\boldsymbol{B} \cdot \boldsymbol{\xi}]\left(\boldsymbol{\xi} \cdot \boldsymbol{\xi}^{\prime}\right) \delta\left[x^{\prime}-\boldsymbol{J} \cdot \boldsymbol{\xi}^{\prime}\right] \delta\left[y^{\prime}-\boldsymbol{B} \cdot \boldsymbol{\xi}^{\prime}\right]\right\rangle_{\tilde{D}}\right\rangle_{\tilde{D}}\right\rangle_{\mathrm{QRP} ; t}$

\section{Batch Learning}

Here we can again use the scaling relation (3.2) to eliminate terms. For $Q$ and $R$ one finds

$$
\begin{gathered}
\frac{d}{d t} Q=\lim _{N \rightarrow \infty}\left\{2 \eta\left\langle\langle(\boldsymbol{J} \cdot \boldsymbol{\xi}) \mathcal{G}[\boldsymbol{J} \cdot \boldsymbol{\xi}, \boldsymbol{B} \cdot \boldsymbol{\xi}]\rangle_{\tilde{D}}\right\rangle_{\mathrm{QRP} ; t}+\frac{\eta^{2}}{N}\left\langle\sum_{i}\left\langle\xi_{i} \mathcal{G}[\boldsymbol{J} \cdot \boldsymbol{\xi}, \boldsymbol{B} \cdot \boldsymbol{\xi}]\right\rangle_{\tilde{D}}^{2}\right\rangle_{\mathrm{QRP} ; t}\right\} \\
=2 \eta \int d x d y P[x, y] x \mathcal{G}[x ; y] \\
\frac{d}{d t} R=\lim _{N \rightarrow \infty} \eta\langle\langle(\boldsymbol{B} \cdot \boldsymbol{\xi}) \\
\left.\mathcal{G}[\boldsymbol{J} \cdot \boldsymbol{\xi}, \boldsymbol{B} \cdot \boldsymbol{\xi}]\rangle_{\tilde{D}}\right\rangle_{\mathrm{QRP} ; t} \\
=\eta \int d x d y P[x, y] y \mathcal{G}[x ; y]
\end{gathered}
$$

Finally we calculate the temporal derivative of the joint field distribution:

$$
\begin{gathered}
\frac{\partial}{\partial t} P[x, y]=\lim _{N \rightarrow \infty}\left\{-\eta \frac{\partial}{\partial x}\left\langle\left\langle\left\langle\mathcal{G}[\boldsymbol{J} \cdot \boldsymbol{\xi}, \boldsymbol{B} \cdot \boldsymbol{\xi}]\left(\boldsymbol{\xi} \cdot \boldsymbol{\xi}^{\prime}\right) \delta\left[x-\boldsymbol{J} \cdot \boldsymbol{\xi}^{\prime}\right] \delta\left[y-\boldsymbol{B} \cdot \boldsymbol{\xi}^{\prime}\right]\right\rangle_{\tilde{D}}\right\rangle_{\tilde{D}}\right\rangle_{\mathrm{QRP} ; t}\right. \\
\left.+\frac{\eta^{2}}{2 N} \frac{\partial^{2}}{\partial x^{2}}\left\langle\sum_{i j}\left\langle\xi_{i} \mathcal{G}[\boldsymbol{J} \cdot \boldsymbol{\xi}, \boldsymbol{B} \cdot \boldsymbol{\xi}]\right\rangle_{\tilde{D}}\left\langle\xi_{j} \mathcal{G}[\boldsymbol{J} \cdot \boldsymbol{\xi}, \boldsymbol{B} \cdot \boldsymbol{\xi}]\right\rangle_{\tilde{D}}\left\langle\xi_{i}^{\prime} \xi_{j}^{\prime} \delta\left[x-\boldsymbol{J} \cdot \boldsymbol{\xi}^{\prime}\right] \delta\left[y-\boldsymbol{B} \cdot \boldsymbol{\xi}^{\prime}\right]\right\rangle_{\tilde{D}}\right\rangle_{\mathrm{QRP} ; t}\right\} \\
=-\eta \frac{\partial}{\partial x} \int d x^{\prime} d y^{\prime} \mathcal{G}\left[x^{\prime}, y^{\prime}\right] \mathcal{C}\left[x, y ; x^{\prime}, y^{\prime}\right]
\end{gathered}
$$

The difference between the macroscopic equations for batch and on-line learning is merely the presence (on-line) or absence (batch) of those terms which are quadratic in the learning rate $\eta$. 


\section{Resume}

We separate the function $\mathcal{C}\left[x, y ; x^{\prime}, y^{\prime}\right](3.6)$, which plays a role similar to that of a Green's function, into two terms $\left(\boldsymbol{\xi}=\boldsymbol{\xi}^{\prime}\right.$ versus $\left.\boldsymbol{\xi} \neq \boldsymbol{\xi}^{\prime}\right)$ :

$$
\begin{aligned}
& \mathcal{C}\left[x, y ; x^{\prime}, y^{\prime}\right]=\alpha^{-1} \delta\left[x-x^{\prime}\right] \delta\left[y-y^{\prime}\right] P[x, y]+\mathcal{A}\left[x, y ; x^{\prime}, y^{\prime}\right] \\
& \mathcal{A}\left[x, y ; x^{\prime}, y^{\prime}\right]=\lim _{N \rightarrow \infty} \\
& \left\langle\left\langle\left\langle\left[1-\delta_{\boldsymbol{\xi}} \boldsymbol{\xi}^{\prime}\right] \delta[x-\boldsymbol{J} \cdot \boldsymbol{\xi}] \delta[y-\boldsymbol{B} \cdot \boldsymbol{\xi}]\left(\boldsymbol{\xi} \cdot \boldsymbol{\xi}^{\prime}\right) \delta\left[x^{\prime}-\boldsymbol{J} \cdot \boldsymbol{\xi}^{\prime}\right] \delta\left[y^{\prime}-\boldsymbol{B} \cdot \boldsymbol{\xi}^{\prime}\right]\right\rangle_{\tilde{D}}\right\rangle_{\tilde{D}}\right\rangle_{\mathrm{ORP} ; t}
\end{aligned}
$$

With this definition our macroscopic laws, which are exact for $N \rightarrow \infty$ but not yet closed due to the appearance of the microscopic probability density $p_{t}(\boldsymbol{J})$ in the sub-shell average of (3.10), can be summarised as follows

$$
\begin{aligned}
& \frac{d}{d t} Q=2 \eta \int d x d y P[x, y] x \mathcal{G}[x ; y]+\kappa \eta^{2} \int d x d y P[x, y] \mathcal{G}^{2}[x ; y] \\
& \frac{d}{d t} R=\eta \int d x d y P[x, y] y \mathcal{G}[x ; y] \\
& \frac{\partial}{\partial t} P[x, y]=-\frac{\eta}{\alpha} \frac{\partial}{\partial x}[\mathcal{G}[x, y] P[x, y]]-\eta \frac{\partial}{\partial x} \int d x^{\prime} d y^{\prime} \mathcal{G}\left[x^{\prime}, y^{\prime}\right] \mathcal{A}\left[x, y ; x^{\prime}, y^{\prime}\right] \\
& \quad+\frac{1}{2} \kappa \eta^{2} \int d x^{\prime} d y^{\prime} P\left[x^{\prime}, y^{\prime}\right] \mathcal{G}^{2}\left[x^{\prime}, y^{\prime}\right] \frac{\partial^{2}}{\partial x^{2}} P[x, y]
\end{aligned}
$$

in which $\kappa=1$ for on-line learning and $\kappa=0$ for batch learning. The complexity of the problem is fully concentrated in the Green's function $\mathcal{A}\left[x, y ; x^{\prime}, y^{\prime}\right]$.

\subsection{Closure of Macroscopic Dynamical Laws}

We now close our macroscopic laws by making, for $N \rightarrow \infty$, the two key assumptions underlying dynamical replica theories:

1. Our macroscopic observables $\{Q, R, P\}$ obey closed dynamic equations.

2. These macroscopic equations are self-averaging with respect to the disorder, i.e. the microscopic realisation of the training set $\tilde{D}$.

Assumption 1 implies that all microscopic probability variations within the $\{Q, R, P\}$ subshells of the $\boldsymbol{J}$-ensemble are either absent or irrelevant to the evolution of $\{Q, R, P\}$. We may consequently make the simplest self-consistent choice for $p_{t}(\boldsymbol{J})$ in evaluating the macroscopic laws, i.e. in (3.10): microscopic probability equipartitioning in the $\{Q, R, P\}$-subshells of the ensemble, or

$$
p_{t}(\boldsymbol{J}) \rightarrow w(\boldsymbol{J}) \sim \delta[Q-Q[\boldsymbol{J}]] \delta[R-R[\boldsymbol{J}]] \prod_{x y} \delta[P[x, y]-P[x, y ; \boldsymbol{J}]]
$$

This distribution depends on time via the order parameters $\{Q, R, P\}$. Note that (3.14) leads to exact macroscopic laws if our observables $\{Q, R, P\}$ for 
$N \rightarrow \infty$ indeed obey closed equations, and is true in equilibrium for detailed balance models in which the Hamiltonian can be written in terms of $\{Q, R, P\}$. It is an approximation if our observables do not obey closed equations. Assumption 2 allows us to average the macroscopic laws over the disorder; for mean-field models it is usually convincingly supported by numerical simulations, and can be proven using the path integral formalism (see e.g. Horner, 1992). We write averages over all training sets $\tilde{D} \subseteq\{-1,1\}^{N}$, with $|\tilde{D}|=p$, as $\langle\ldots\rangle$. Our assumptions result in the closure of (3.11,3.12,3.13), since now the function $\mathcal{A}\left[x, y ; x^{\prime}, y^{\prime}\right]$ is expressed fully in terms of $\{Q, R, P\}$ :

$$
\begin{gathered}
\mathcal{A}\left[x, y ; x^{\prime}, y^{\prime}\right]=\lim _{N \rightarrow \infty} \\
\left\langle\frac{\int d \boldsymbol{J} w(\boldsymbol{J})\left\langle\left\langle\delta[x-\boldsymbol{J} \cdot \boldsymbol{\xi}] \delta[y-\boldsymbol{B} \cdot \boldsymbol{\xi}]\left(\boldsymbol{\xi} \cdot \boldsymbol{\xi}^{\prime}\right)\left[1-\delta \boldsymbol{\xi}^{\prime}\right] \delta\left[x^{\prime}-\boldsymbol{J} \cdot \boldsymbol{\xi}^{\prime}\right] \delta\left[y^{\prime}-\boldsymbol{B} \cdot \boldsymbol{\xi}^{\prime}\right]\right\rangle_{\tilde{D}}\right\rangle_{\tilde{D}}}{\int d \boldsymbol{J} w(\boldsymbol{J})}\right\rangle_{\boldsymbol{\Xi}}
\end{gathered}
$$

The final ingredient of dynamical replica theory is the realization that averages of fractions can be calculated with the replica identity

$$
\left\langle\frac{\int d \boldsymbol{J} W[\boldsymbol{J}, \boldsymbol{z}] G[\boldsymbol{J}, \boldsymbol{z}]}{\int d \boldsymbol{J} W[\boldsymbol{J}, \boldsymbol{z}]}\right\rangle_{\boldsymbol{z}}=\lim _{n \rightarrow 0} \int d \boldsymbol{J}^{1} \cdots d \boldsymbol{J}^{n}\left\langle G\left[\boldsymbol{J}^{1}, \boldsymbol{z}\right] \prod_{\alpha=1}^{n} W\left[\boldsymbol{J}^{\alpha}, \boldsymbol{z}\right]\right\rangle \boldsymbol{z}
$$

giving

$$
\begin{aligned}
& \mathcal{A}\left[x, y ; x^{\prime}, y^{\prime}\right]=\lim _{N \rightarrow \infty} \lim _{n \rightarrow 0} \int \prod_{\alpha=1}^{n} w\left(\boldsymbol{J}^{\alpha}\right) d \boldsymbol{J}^{\alpha} \\
& \left\langle\left\langle\left\langle\delta\left[x-\boldsymbol{J}^{1} \cdot \boldsymbol{\xi}\right] \delta[y-\boldsymbol{B} \cdot \boldsymbol{\xi}]\left(\boldsymbol{\xi} \cdot \boldsymbol{\xi}^{\prime}\right)\left[1-\delta_{\boldsymbol{\xi} \boldsymbol{\xi}^{\prime}}\right] \delta\left[x^{\prime}-\boldsymbol{J}^{1} \cdot \boldsymbol{\xi}^{\prime}\right] \delta\left[y^{\prime}-\boldsymbol{B} \cdot \boldsymbol{\xi}^{\prime}\right]\right\rangle_{\tilde{D}}\right\rangle_{\tilde{D}}\right\rangle_{\mathbf{\Xi}}
\end{aligned}
$$

Since each weight component scales as $J_{i}^{\alpha}=\mathcal{O}\left(N^{-\frac{1}{2}}\right)$ we transform variables in such a way that our calculations will involve $\mathcal{O}(1)$ objects:

$$
(\forall i)(\forall \alpha): \quad J_{i}^{\alpha}=(Q / N)^{\frac{1}{2}} \sigma_{i}^{\alpha}, \quad B_{i}=N^{-\frac{1}{2}} \tau_{i}
$$

This ensures $\sigma_{i}^{\alpha}=\mathcal{O}(1), \tau_{i}=\mathcal{O}(1)$, and reduces various constraints to ordinary spherical ones: $\left(\boldsymbol{\sigma}^{\alpha}\right)^{2}=\boldsymbol{\tau}^{2}=N$ for all $\alpha$. Overall prefactors generated by these transformations always vanish due to $n \rightarrow 0$. We find a new effective measure: $\prod_{\alpha=1}^{n} w\left(\boldsymbol{J}^{\alpha}\right) d \boldsymbol{J}^{\alpha} \rightarrow \prod_{\alpha=1}^{n} \tilde{w}\left(\boldsymbol{\sigma}^{\alpha}\right) d \boldsymbol{\sigma}^{\alpha}$, with

$$
\tilde{w}(\boldsymbol{\sigma}) \sim \delta\left[N-\boldsymbol{\sigma}^{2}\right] \delta\left[N R Q^{-\frac{1}{2}}-\boldsymbol{\tau} \cdot \boldsymbol{\sigma}\right] \prod_{x y} \delta\left[P[x, y]-P\left[x, y ;(Q / N)^{\frac{1}{2}} \boldsymbol{\sigma}\right]\right]
$$

We thus arrive at

$$
\begin{aligned}
& \mathcal{A}\left[x, y ; x^{\prime}, y^{\prime}\right]=\lim _{n \rightarrow 0} \lim _{N \rightarrow \infty} \int \prod_{\alpha=1}^{n} \tilde{w}\left(\boldsymbol{\sigma}^{\alpha}\right) d \boldsymbol{\sigma}^{\alpha}\left\langle\left\langle\left(\boldsymbol{\xi}^{\prime} \cdot \boldsymbol{\xi}\right)\left[1-\delta_{\boldsymbol{\xi} \boldsymbol{\xi}^{\prime}}\right]\right.\right. \\
& \left.\left.\left.\delta\left[x-\frac{\sqrt{Q} \boldsymbol{\sigma}^{1} \cdot \boldsymbol{\xi}}{\sqrt{N}}\right] \delta\left[y-\frac{\boldsymbol{\tau} \cdot \boldsymbol{\xi}}{\sqrt{N}}\right] \delta\left[x^{\prime}-\frac{\sqrt{Q} \boldsymbol{\sigma}^{1} \cdot \boldsymbol{\xi}^{\prime}}{\sqrt{N}}\right] \delta\left[y-\frac{\boldsymbol{\tau} \cdot \boldsymbol{\xi}^{\prime}}{\sqrt{N}}\right]\right\rangle_{\tilde{D}}\right\rangle_{\tilde{D}}\right\rangle_{\boldsymbol{\Xi}}
\end{aligned}
$$


In the same fashion one can also express $P[x, y]$ in replica form (which will prove useful for normalization purposes and for self-consistency tests):

$$
P_{t}[x, y]=\lim _{n \rightarrow 0} \lim _{N \rightarrow \infty} \int \prod_{\alpha=1}^{n} \tilde{w}\left(\boldsymbol{\sigma}^{\alpha}\right) d \boldsymbol{\sigma}^{\alpha}\left\langle\left\langle\delta\left[x-\frac{\sqrt{Q} \boldsymbol{\sigma}^{1} \cdot \boldsymbol{\xi}}{\sqrt{N}}\right] \delta\left[y-\frac{\boldsymbol{\tau} \cdot \boldsymbol{\xi}}{\sqrt{N}}\right]\right\rangle_{\tilde{D}}\right\rangle_{\boldsymbol{\Xi}}
$$

\section{Replica Calculation of the Green's Function}

\subsection{Disorder Averaging}

In order to perform the disorder average we insert integral representations for the $\delta$-functions which define the fields $\left(x, y, x^{\prime}, y^{\prime}\right)$ and for the $\delta$-functions in the measure (3.15) which involve $P[x, y]$, generating $n$ conjugate order parameter functions $\hat{P}_{\alpha}(x, y)$. Upon also writing averages over the training set in terms of the $p$ constituent vectors $\left\{\boldsymbol{\xi}^{\mu}\right\}$ we obtain for (3.16) and (3.17):

$$
\begin{gathered}
\mathcal{A}\left[x, y ; x^{\prime}, y^{\prime}\right]=\int \frac{d \hat{x} d \hat{x}^{\prime} d \hat{y} d \hat{y}^{\prime}}{(2 \pi)^{4}} e^{i\left[x \hat{x}+x^{\prime} \hat{x}^{\prime}+y \hat{y}+y \hat{y}^{\prime}\right]} \lim _{n \rightarrow 0} \lim _{N \rightarrow \infty} \int \prod_{\alpha=1}^{n} \prod_{x^{\prime \prime} y^{\prime \prime}} d \hat{P}_{\alpha}\left(x^{\prime \prime}, y^{\prime \prime}\right) \\
\int \prod_{\alpha=1}^{n}\left\{d \boldsymbol{\sigma}^{\alpha} \delta\left[N-\left(\boldsymbol{\sigma}^{\alpha}\right)^{2}\right] \delta\left[\frac{N R}{\sqrt{Q}}-\boldsymbol{\tau} \cdot \boldsymbol{\sigma}^{\alpha}\right] e^{i N \int d x^{\prime \prime} d y^{\prime \prime} \hat{P}_{\alpha}\left(x^{\prime \prime}, y^{\prime \prime}\right) P_{t}\left(x^{\prime \prime}, y^{\prime \prime}\right)}\right\} \\
\left\langle\frac{1}{p^{2}} \sum_{\mu \neq \nu}\left(\boldsymbol{\xi}^{\mu} \cdot \boldsymbol{\xi}^{\nu}\right) e^{-\frac{i}{\alpha} \sum_{\alpha \lambda} \hat{P}_{\alpha}\left(\frac{\sqrt{Q} \boldsymbol{\sigma}^{\alpha} \cdot \boldsymbol{\xi}^{\lambda}}{\sqrt{N}}, \frac{\boldsymbol{\tau} \cdot \boldsymbol{\xi}^{\lambda}}{\sqrt{N}}\right)-\frac{i}{\sqrt{N}} \boldsymbol{\xi}^{\mu} \cdot\left[\hat{x} \sqrt{Q} \boldsymbol{\sigma}^{1}+\hat{y} \boldsymbol{\tau}\right]-\frac{i}{\sqrt{N}} \boldsymbol{\xi}^{\nu} \cdot\left[\hat{x}^{\prime} \sqrt{Q} \boldsymbol{\sigma}^{1}+\hat{y}^{\prime} \boldsymbol{\tau}\right]}\right\rangle_{\boldsymbol{\Xi}} \\
P[x, y]=\int \frac{d \hat{x} d \hat{y}}{(2 \pi)^{2}} e^{i[x \hat{x}+y \hat{y}]} \lim _{n \rightarrow 0} \lim _{N \rightarrow \infty} \int \prod_{\alpha=1}^{n} \prod_{x^{\prime \prime} y^{\prime \prime}} d \hat{P}_{\alpha}\left(x^{\prime \prime}, y^{\prime \prime}\right) \\
\int \prod_{\alpha=1}^{n}\left\{d \boldsymbol{\sigma}^{\alpha} \delta\left[N-\left(\boldsymbol{\sigma}^{\alpha}\right)^{2}\right] \delta\left[\frac{N R}{\sqrt{Q}}-\boldsymbol{\tau} \cdot \boldsymbol{\sigma}^{\alpha}\right] e^{i N \int d x^{\prime \prime} d y^{\prime \prime} \hat{P}_{\alpha}\left(x^{\prime \prime}, y^{\prime \prime}\right) P_{t}\left(x^{\prime \prime}, y^{\prime \prime}\right)}\right\} \\
\left\langle\frac{1}{p} \sum_{\mu=1}^{p} e^{\left.-\frac{i}{\alpha} \sum_{\alpha \lambda} \hat{P}_{\alpha}\left(\frac{\sqrt{Q} \boldsymbol{\sigma} \boldsymbol{\sigma}^{\alpha}}{\sqrt{N}}, \frac{\boldsymbol{\tau} \cdot \boldsymbol{\xi}^{\lambda}}{\sqrt{N}}\right)-\frac{i}{\sqrt{N}} \boldsymbol{\xi}^{\mu} \cdot \hat{x} \sqrt{Q} \boldsymbol{\sigma}^{1}+\hat{y} \boldsymbol{\tau}\right]}\right\rangle_{\boldsymbol{\Xi}}
\end{gathered}
$$

In calculating the averages over the training sets $\langle\ldots\rangle \Xi$ that occur in (4.1) and (4.2) one can use permutation symmetries with respect to sites and pattern labels, leading to the following compact results:

$$
\begin{gathered}
\left\langle\frac{1}{p^{2}} \sum_{\mu \neq \nu}\left(\boldsymbol{\xi}^{\mu} \boldsymbol{\xi}^{\nu}\right) e^{\left.-\frac{i}{\alpha} \sum_{\alpha} \sum_{\lambda} \hat{P}_{\alpha}\left(\frac{\sqrt{Q} \boldsymbol{\sigma}^{\alpha} \cdot \boldsymbol{\xi}^{\lambda}}{\sqrt{N}}, \frac{\boldsymbol{\tau} \cdot \boldsymbol{\xi}^{\lambda}}{\sqrt{N}}\right)-\frac{i}{\sqrt{N}} \boldsymbol{\xi}^{\mu} \cdot \hat{x} \sqrt{Q} \boldsymbol{\sigma}^{1}+\hat{y} \boldsymbol{\tau}\right]-\frac{i}{\sqrt{N}} \boldsymbol{\xi}^{\nu} \cdot\left[\hat{x}^{\prime} \sqrt{Q} \boldsymbol{\sigma}^{1}+\hat{y}^{\prime} \boldsymbol{\tau}\right]}\right\rangle_{\boldsymbol{\Xi}} \\
=e^{p \log \mathcal{D}[0,0]} \frac{1}{N} \sum_{j} \frac{\mathcal{E}_{j}[\hat{x}, \hat{y}] \mathcal{E}_{j}\left[\hat{x}^{\prime}, \hat{y}^{\prime}\right]}{\mathcal{D}^{2}[0,0]}+\mathcal{O}\left(N^{-\frac{1}{2}}\right)
\end{gathered}
$$


and

$$
\begin{gathered}
\left\langle\frac{1}{p} \sum_{\mu=1}^{p} e^{\left.-\frac{i}{\alpha} \sum_{\alpha} \sum_{\lambda} \hat{P}_{\alpha}\left(\frac{\sqrt{Q} \boldsymbol{\sigma}^{\alpha} \cdot \boldsymbol{\xi}^{\lambda}}{\sqrt{N}}, \frac{\boldsymbol{\tau} \cdot \boldsymbol{\xi}^{\lambda}}{\sqrt{N}}\right)-\frac{i}{\sqrt{N}} \boldsymbol{\xi}^{\mu} \cdot \hat{x} \sqrt{Q} \boldsymbol{\sigma}^{1}+\hat{y} \boldsymbol{\tau}\right]}\right\rangle_{\boldsymbol{\Xi}} \\
=e^{p \log \mathcal{D}[0,0]} \frac{\mathcal{D}[\hat{x}, \hat{y}]}{\mathcal{D}[0,0]}+\mathcal{O}\left(N^{-\frac{1}{2}}\right)
\end{gathered}
$$

in which

$$
\begin{aligned}
& \mathcal{D}[u, v]=\left\langle e^{-\frac{i}{\alpha} \sum_{\alpha} \hat{P}_{\alpha}\left(\frac{\sqrt{Q} \boldsymbol{\sigma}^{\alpha} \cdot \boldsymbol{\xi}}{\sqrt{N}}, \frac{\boldsymbol{\tau} \cdot \boldsymbol{\xi}}{\sqrt{N}}\right)-\frac{i}{\sqrt{N}} \boldsymbol{\xi} \cdot\left[u \sqrt{Q} \boldsymbol{\sigma}^{1}+v \boldsymbol{\tau}\right]}\right\rangle \boldsymbol{\xi} \\
& \mathcal{E}_{j}[u, v]=\left\langle\sqrt{N} \xi_{j} e^{-\frac{i}{\alpha} \sum_{\alpha} \hat{P}_{\alpha}\left(\frac{\sqrt{Q} \boldsymbol{\sigma}^{\alpha} \cdot \boldsymbol{\xi}}{\sqrt{N}}, \frac{\boldsymbol{\tau} \cdot \boldsymbol{\xi}}{\sqrt{N}}\right)-\frac{i}{\sqrt{N}} \boldsymbol{\xi} \cdot\left[u \sqrt{Q} \boldsymbol{\sigma}^{1}+v \boldsymbol{\tau}\right]}\right\rangle_{\boldsymbol{\xi}}
\end{aligned}
$$

and with the abbreviation $\langle f[\boldsymbol{\xi}]\rangle_{\boldsymbol{\xi}}=2^{-N} \sum_{\boldsymbol{\xi} \in\{-1,1\}^{N}} f[\boldsymbol{\xi}]$. These quantities (which are both $\mathcal{O}(1)$ for $N \rightarrow \infty$ ) are, in turn, evaluated by using the central limit theorem, which ensures that for $N \rightarrow \infty$ the $n$ rescaled inner products $\boldsymbol{\sigma}^{\alpha} \cdot \boldsymbol{\xi} / \sqrt{N}$ and the rescaled inner product $\boldsymbol{\tau} \cdot \boldsymbol{\xi} / \sqrt{N}$ will become (correlated) zero-average Gaussian variables. After some algebra one finds

$$
\begin{aligned}
& \mathcal{L}\left[u, v ; u^{\prime}, v^{\prime}\right]=\frac{1}{N} \sum_{j} \mathcal{E}_{j}[u, v] \mathcal{E}_{j}\left[u^{\prime}, v^{\prime}\right]= \\
& =-Q \sum_{\alpha \beta} q_{\alpha \beta}(\{\boldsymbol{\sigma}\})\left[\frac{1}{\alpha} \mathcal{F}_{1}^{\alpha}[u, v]+u \delta_{\alpha 1} \mathcal{D}[u, v]\right]\left[\frac{1}{\alpha} \mathcal{F}_{1}^{\beta}\left[u^{\prime}, v^{\prime}\right]+u^{\prime} \delta_{\beta 1} \mathcal{D}\left[u^{\prime}, v^{\prime}\right]\right] \\
& \quad-R \sum_{\alpha \beta}\left[\frac{1}{\alpha} \mathcal{F}_{1}^{\alpha}[u, v]+u \delta_{\alpha 1} \mathcal{D}[u, v]\right]\left[\frac{1}{\alpha} \mathcal{F}_{2}^{\beta}\left[u^{\prime}, v^{\prime}\right]+v^{\prime} \delta_{\beta 1} \mathcal{D}\left[u^{\prime}, v^{\prime}\right]\right] \\
& \quad-R \sum_{\alpha \beta}\left[\frac{1}{\alpha} \mathcal{F}_{1}^{\alpha}\left[u^{\prime}, v^{\prime}\right]+u^{\prime} \delta_{\alpha 1} \mathcal{D}\left[u^{\prime}, v^{\prime}\right]\right]\left[\frac{1}{\alpha} \mathcal{F}_{2}^{\beta}[u, v]+v \delta_{\beta 1} \mathcal{D}[u, v]\right] \\
& -\sum_{\alpha \beta}\left[\frac{1}{\alpha} \mathcal{F}_{2}^{\alpha}[u, v]+v \delta_{\alpha 1} \mathcal{D}[u, v]\right]\left[\frac{1}{\alpha} \mathcal{F}_{2}^{\beta}\left[u^{\prime}, v^{\prime}\right]+v^{\prime} \delta_{\beta 1} \mathcal{D}\left[u^{\prime}, v^{\prime}\right]\right]+\mathcal{O}\left(N^{-\frac{1}{2}}\right)
\end{aligned}
$$

in which $\mathcal{D}[u, v]$ and the $\mathcal{F}_{\lambda}^{\alpha}[u, v]$ are given by $n+1$ dimensional integrals:

$$
\begin{aligned}
& \mathcal{D}[u, v]=\int \frac{d \boldsymbol{x} d y \operatorname{det}^{\frac{1}{2}} \boldsymbol{A}}{(2 \pi)^{(n+1) / 2}} e^{-\frac{1}{2}\left(\begin{array}{c}
\boldsymbol{x} \\
y
\end{array}\right) \cdot \boldsymbol{A}\left(\begin{array}{l}
\boldsymbol{x} \\
y
\end{array}\right)-\frac{i}{\alpha} \sum_{\alpha} \hat{P}_{\alpha}\left(\sqrt{Q} x_{\alpha}, y\right)-i\left[u \sqrt{Q} x_{1}+v y\right]} \\
& \mathcal{F}_{\lambda}^{\alpha}[u, v]= \\
& \int \frac{d \boldsymbol{x} d y \operatorname{det}^{\frac{1}{2}} \boldsymbol{A}}{(2 \pi)^{(n+1) / 2}} \partial_{\lambda} \hat{P}_{\alpha}\left(\sqrt{Q} x_{\alpha}, y\right) e^{-\frac{1}{2}\left(\begin{array}{c}
\boldsymbol{x} \\
y
\end{array}\right) \cdot \boldsymbol{A}\left(\begin{array}{c}
\boldsymbol{x} \\
y
\end{array}\right)-\frac{i}{\alpha} \sum_{\alpha} \hat{P}_{\alpha}\left(\sqrt{Q} x_{\alpha}, y\right)-i\left[u \sqrt{Q} x_{1}+v y\right]}
\end{aligned}
$$


with $\lambda \in\{1,2\}$. The matrix $\boldsymbol{A}$ in (4.6,4.7) is defined by

$$
\boldsymbol{A}^{-1}=\left(\begin{array}{cccc}
q_{11} & \cdots & q_{1 n} & R / \sqrt{Q} \\
\vdots & & \vdots & \vdots \\
q_{n 1} & \cdots & q_{n n} & R / \sqrt{Q} \\
R / \sqrt{Q} & \cdots & R / \sqrt{Q} & 1
\end{array}\right) \quad q_{\alpha \beta}(\{\boldsymbol{\sigma}\})=\frac{1}{N} \sum_{i} \sigma_{i}^{\alpha} \sigma_{i}^{\beta}
$$

Note that the quantities (4.6,4.7) depend on the microscopic variables $\boldsymbol{\sigma}^{\alpha}$ only through the spin-glass order parameters $q_{\alpha \beta}(\{\boldsymbol{\sigma}\})$.

\subsection{Derivation of Saddle-Point Equations}

We combine the results $(4.3,4.4,4.5)$ with $(4.1,4.2)$. We use integral representations for the remaining $\delta$-functions, and isolate the $q_{\alpha \beta}$, by inserting

$$
\begin{aligned}
1=\int \frac{d \boldsymbol{q} d \hat{\boldsymbol{q}} d \hat{\boldsymbol{Q}} d \hat{\boldsymbol{R}}}{(2 \pi / N)^{n^{2}+2 n}} e^{i N\left[\sum_{\alpha}\left(\hat{Q}_{\alpha}+\hat{R}_{\alpha} R / \sqrt{Q}\right)+\sum_{\alpha \beta} \hat{q}_{\alpha \beta} q_{\alpha \beta}\right]} & \\
& \times e^{-i \sum_{i}\left[\sum_{\alpha}\left(\hat{Q}_{\alpha}\left(\sigma_{i}^{\alpha}\right)^{2}+\hat{R}_{\alpha} \tau_{i} \sigma_{i}^{\alpha}\right)-i \sum_{\alpha \beta} \hat{q}_{\alpha \beta} \sigma_{i}^{\alpha} \sigma_{i}^{\beta}\right]}
\end{aligned}
$$

We hereby achieve a full factorisation over sites, and both (4.1) and (4.2) can be written in the form of an integral dominated by saddle-points:

$$
\begin{aligned}
& \mathcal{A}\left[x, y ; x^{\prime}, y^{\prime}\right]=\int \frac{d \hat{x} d \hat{x}^{\prime} d \hat{y} d \hat{y}^{\prime}}{(2 \pi)^{4}} e^{i\left[x \hat{x}+x^{\prime} \hat{x}^{\prime}+y \hat{y}+y \hat{y}^{\prime}\right]} \\
& \lim _{n \rightarrow 0} \lim _{N \rightarrow \infty} \int d \boldsymbol{q} d \hat{\boldsymbol{q}} d \hat{\boldsymbol{Q}} d \hat{\boldsymbol{R}} \prod_{\alpha x^{\prime \prime} y^{\prime \prime}} d \hat{P}_{\alpha}\left(x^{\prime \prime}, y^{\prime \prime}\right) e^{N \Psi[\boldsymbol{q}, \hat{\boldsymbol{q}}, \hat{\boldsymbol{Q}}, \hat{\boldsymbol{R}},\{\hat{P}\}]} \frac{\mathcal{L}\left[\hat{x}, \hat{y} ; \hat{x}^{\prime}, \hat{y}^{\prime}\right]}{\mathcal{D}^{2}[0,0]} \\
& P[x, y]=\int \frac{d \hat{x} d \hat{y}}{(2 \pi)^{2}} e^{i[x \hat{x}+y \hat{y}]} \\
& \lim _{n \rightarrow 0} \lim _{N \rightarrow \infty} \int d \boldsymbol{q} d \hat{\boldsymbol{q}} d \hat{\boldsymbol{Q}} d \hat{\boldsymbol{R}} \prod_{\alpha x^{\prime \prime} y^{\prime \prime}} d \hat{P}_{\alpha}\left(x^{\prime \prime}, y^{\prime \prime}\right) e^{N \Psi[\boldsymbol{q}, \hat{\boldsymbol{q}}, \hat{\boldsymbol{Q}}, \hat{\boldsymbol{R}},\{\hat{P}\}]} \frac{\mathcal{D}[\hat{x}, \hat{y}]}{\mathcal{D}[0,0]}
\end{aligned}
$$

with

$$
\begin{gathered}
\Psi[\ldots]=i \sum_{\alpha}\left(\hat{Q}_{\alpha}+\hat{R}_{\alpha} R / \sqrt{Q}\right)+i \sum_{\alpha \beta} \hat{q}_{\alpha \beta} q_{\alpha \beta}+i \sum_{\alpha} \int d x d y \hat{P}_{\alpha}(x, y) P[x, y] \\
+\alpha \log \mathcal{D}[0,0]+\lim _{N \rightarrow \infty} \frac{1}{N} \sum_{i} \log \int d \boldsymbol{\sigma} e^{-i \sum_{\alpha}\left[\hat{Q}_{\alpha} \sigma_{\alpha}^{2}+\hat{R}_{\alpha} \tau_{i} \sigma_{\alpha}\right]-i \sum_{\alpha \beta} \hat{q}_{\alpha \beta} \sigma_{\alpha} \sigma_{\beta}}
\end{gathered}
$$

The above expressions for $\mathcal{A}\left[x, y ; x^{\prime}, y^{\prime}\right]$ and $P[x, y]$ will be given by the intensive parts of the integrands, evaluated in the dominating saddle-point of $\Psi$. We can use the equation for $P[x, y]$ to verify that all expressions are properly normalized. After a simple transformation of some integration variables,

$$
\hat{q}_{\alpha \beta} \rightarrow \hat{q}_{\alpha \beta}-\hat{Q}_{\alpha} \delta_{\alpha \beta} \quad \hat{R}_{\alpha} \rightarrow \sqrt{Q} \hat{R}_{\alpha}
$$


we arrive at the simple result

$$
\begin{aligned}
\mathcal{A}\left[x, y ; x^{\prime}, y^{\prime}\right] & =\int \frac{d \hat{x} d \hat{x}^{\prime} d \hat{y} d \hat{y}^{\prime}}{(2 \pi)^{4}} e^{i\left[x \hat{x}+x^{\prime} \hat{x}^{\prime}+y \hat{y}+y \hat{y}^{\prime}\right]} \lim _{n \rightarrow 0} \frac{\mathcal{L}\left[\hat{x}, \hat{y} ; \hat{x}^{\prime}, \hat{y}^{\prime}\right]}{\mathcal{D}^{2}[0,0]} \\
P[x, y] & =\int \frac{d \hat{x} d \hat{y}}{(2 \pi)^{2}} e^{i[x \hat{x}+y \hat{y}]} \lim _{n \rightarrow 0} \frac{\mathcal{D}[\hat{x}, \hat{y}]}{\mathcal{D}[0,0]}
\end{aligned}
$$

in which all functions are to be evaluated upon choosing for the order parameters the appropriate saddle-point of $\Psi$, which itself takes the form:

$$
\begin{gathered}
\Psi[\ldots]=i \sum_{\alpha} \hat{Q}_{\alpha}\left(1-q_{\alpha \alpha}\right)+i R \sum_{\alpha} \hat{R}_{\alpha}+i \sum_{\alpha \beta} \hat{q}_{\alpha \beta} q_{\alpha \beta}+i \sum_{\alpha} \int d x d y \hat{P}_{\alpha}(x, y) P[x, y] \\
+\alpha \log \mathcal{D}[0,0]+\lim _{N \rightarrow \infty} \frac{1}{N} \sum_{i} \log \int d \boldsymbol{\sigma} e^{-i \tau_{i} \sqrt{Q} \sum_{\alpha} \hat{R}_{\alpha} \sigma_{\alpha}-i \sum_{\alpha \beta} \hat{q}_{\alpha \beta} \sigma_{\alpha} \sigma_{\beta}}
\end{gathered}
$$

With $\mathcal{D}[u, v]$ given by (4.6) and with the function $\mathcal{L}\left[u, v ; u^{\prime}, v^{\prime}\right]$ given by (4.5). The auxiliary order parameters $q_{\alpha \beta}$ have the usual interpretation in terms of the average probability density for finding a mutual overlap $q$ of two independently evolving weight vectors with the same realization of the training set (see e.g. Mézard et al, 1987):

$$
\langle P(q)\rangle_{\boldsymbol{\Xi}}=\left\langle\left\langle\left\langle\delta\left[q-\frac{\boldsymbol{J}^{a} \cdot \boldsymbol{J}^{b}}{\left|\boldsymbol{J}^{a}\right|\left|\boldsymbol{J}^{b}\right|}\right]\right\rangle\right\rangle_{\boldsymbol{\Xi}}=\lim _{n \rightarrow 0} \frac{1}{n(n-1)} \sum_{\alpha \neq \beta} \delta\left[q-q_{\alpha \beta}\right]\right.
$$

We now make the replica symmetric (RS) ansatz in the extremisation problem, which according to (4.12) is equivalent to assuming ergodicity. With a modest amount of foresight we put

$$
\begin{array}{lll}
q_{\alpha \beta}=q_{0} \delta_{\alpha \beta}+q\left[1-\delta_{\alpha \beta}\right] & \hat{q}_{\alpha \beta}=\frac{i}{2}\left[r-r_{0} \delta_{\alpha \beta}\right] \\
\hat{R}_{\alpha}=i \rho & \hat{Q}_{\alpha}=i \phi & \hat{P}_{\alpha}(u, v)=i \chi[u, v]
\end{array}
$$

This allows us to expand the quantity $\Psi$ of (4.11) for small $n$ :

$$
\begin{gathered}
\lim _{n \rightarrow 0} \frac{1}{n} \Psi[\ldots]=-\phi\left(1-q_{0}\right)-\rho R+\frac{1}{2} q r-\frac{1}{2} q_{0}\left(r-r_{0}\right)-\frac{1}{2} \log r_{0}+\frac{1}{2 r_{0}}\left(r+\rho^{2} Q\right) \\
-\int d x d y \chi[x, y] P[x, y]+\lim _{n \rightarrow 0} \frac{\alpha}{n} \log \mathcal{D}[0,0]+\text { constants }
\end{gathered}
$$

At this stage it is useful to work out those saddle-point equations that follow upon variation of $\left\{\phi, r, \rho, r_{0}\right\}$ :

$$
q_{0}=1 \quad r_{0}=\frac{1}{1-q} \quad \rho=\frac{R}{Q(1-q)} \quad r=\frac{q Q-R^{2}}{Q(1-q)^{2}}
$$


These allow us to eliminate most variational parameters, leaving a saddlepoint problem involving only the function $\chi[x, y]$ and the scalar $q$ :

$$
\begin{gathered}
\lim _{n \rightarrow 0} \frac{1}{n} \Psi[q,\{\chi\}]=\frac{1-R^{2} / Q}{2(1-q)}+\frac{1}{2} \log (1-q)-\int d x d y \chi[x, y] P[x, y] \\
+\lim _{n \rightarrow 0} \frac{\alpha}{n} \log \mathcal{D}[0,0 ; q,\{\chi\}]+\text { constants }
\end{gathered}
$$

Finally we have to work out the $\mathrm{RS}$ version of $\mathcal{D}[0,0 ; q,\{\chi\}]$, as defined more generally in (4.6). The inverse of the matrix in (4.8), in RS ansatz, is found to be:

$$
\boldsymbol{A}=\left(\begin{array}{cccc}
C_{11} & \cdots & C_{1 n} & \gamma \\
\vdots & & \vdots & \vdots \\
C_{n 1} & \cdots & C_{n n} & \gamma \\
\gamma & \cdots & \gamma & b
\end{array}\right) \quad C_{\alpha \beta}=\frac{\delta_{\alpha \beta}}{1-q}-d \quad \begin{aligned}
& \gamma=-\frac{R / \sqrt{Q}}{1-q}+\mathcal{O}(n) \\
&
\end{aligned}
$$

With this expression we obtain

$$
\begin{gathered}
\mathcal{D}[0,0 ; q,\{\chi\}]=\frac{\int d \boldsymbol{x} d y e^{-\frac{1}{2} \boldsymbol{x} \cdot \boldsymbol{C} \boldsymbol{x}-\frac{1}{2} b y^{2}-\gamma y \sum_{\alpha} x_{\alpha}+\frac{1}{\alpha} \sum_{\alpha} \chi\left(\sqrt{Q} x_{\alpha}, y\right)}}{\int d \boldsymbol{x} d y e^{-\frac{1}{2} \boldsymbol{x} \cdot \boldsymbol{C} \boldsymbol{x}-\frac{1}{2} b y^{2}-\gamma y \sum_{\alpha} x_{\alpha}}} \\
=\frac{\int D z D y\left[\int d x e^{-\frac{x^{2}}{2(1-q)}+\left[z \sqrt{d}-\gamma \frac{y}{\sqrt{b}}\right] x+\frac{1}{\alpha} \chi\left(\sqrt{Q} x, \frac{y}{\sqrt{b}}\right)}\right]^{n}}{\int D z D y\left[\int d x e^{-\frac{1}{2(1-q)} x^{2}+\left[z \sqrt{d}-\gamma \frac{y}{\sqrt{b}}\right] x}\right]^{n}} \\
\lim _{n \rightarrow 0} \frac{\alpha}{n} \log \mathcal{D}[0,0 ; q,\{\chi\}]=\alpha \int D z D y \log \left\{\frac{\int d x e^{-\frac{x^{2}}{2 Q(1-q)}+x[z \sqrt{d}-\gamma y] / \sqrt{Q}+\frac{1}{\alpha} \chi(x, y)}}{\int d x e^{-\frac{x^{2}}{2 Q(1-q)}+x[z \sqrt{d}-\gamma y] / \sqrt{Q}}}\right\}
\end{gathered}
$$

We can simplify this result by defining

$$
A=R / Q(1-q) \quad B=\sqrt{q Q-R^{2}} / Q(1-q)
$$

which gives

$$
\lim _{n \rightarrow 0} \frac{\alpha}{n} \log \mathcal{D}[0,0 ; q,\{\chi\}]=\alpha \int D z D y \log \left\{\frac{\int d x e^{-\frac{x^{2}}{2 Q(1-q)}+x[A y+B z]+\frac{1}{\alpha} \chi(x, y)}}{\int d x e^{-\frac{x^{2}}{2 Q(1-q)}+x[A y+B z]}}\right\}
$$

Upon carrying out the $x$-integration in the denominator of this expression we can now write (4.13) in a surprisingly simple form (with the short-hand (4.15)):

$\lim _{n \rightarrow 0} \frac{1}{n} \Psi[q,\{\chi\}]=\frac{1-\alpha-R^{2} / Q}{2(1-q)}+\frac{1}{2}(1-\alpha) \log (1-q)-\int d x d y \chi[x, y] P[x, y]$ 


$$
+\alpha \int D z D y \log \int d x e^{-\frac{x^{2}}{2 Q(1-q)}+x[A y+B z]+\frac{1}{\alpha} \chi[x, y]}
$$

Note that (4.16) is to be minimised, both with respect to $q$ (which originated as an $n(n-1)$-fold entry in a matrix, leading to curvature sign change for $n<1$ ) and with respect to $\chi[x, y]$ (obtained from the $n$-fold occurrence of the function $\hat{P}[x, y]$, multiplied by $i$, which also leads to a curvature sign change).

The remaining saddle point equations correspond to the (functional) variation with respect to $\chi$ :

$$
\text { for all } x, y: \quad P[x, y]=\frac{e^{-\frac{1}{2} y^{2}}}{\sqrt{2 \pi}} \int D z\left\{\frac{e^{-\frac{x^{2}}{2 Q(1-q)}+x[A y+B z]+\frac{1}{\alpha} \chi[x, y]}}{\int d x^{\prime} e^{-\frac{x^{\prime 2}}{2 Q(1-q)}+x^{\prime}[A y+B z]+\frac{1}{\alpha} \chi\left[x^{\prime}, y\right]}}\right\} \text {, }
$$

and $q$ (using equation (4.17) wherever possible):

$$
\begin{gathered}
\int d x d y P[x, y] x^{2}-2 R \int d x d y P[x, y] x y-q Q\left(\alpha^{-1}-1\right)+R^{2} \alpha^{-1} \\
=\left[2 \sqrt{q Q-R^{2}}+\frac{Q(1-q)}{\sqrt{q Q-R^{2}}}\right] \int D z D y \frac{\partial}{\partial z}\left\{\frac{\int d x x e^{-\frac{x^{2}}{2 Q(1-q)}+x[A y+B z]+\frac{1}{\alpha} \chi[x, y]}}{\int d x e^{-\frac{x^{2}}{2 Q(1-q)}+x[A y+B z]+\frac{1}{\alpha} \chi[x, y]}}\right\}
\end{gathered}
$$

\subsection{Explicit Expression for the Green's Function}

In order to work out the Green's function (4.9) we need $\mathcal{L}\left[u, v ; u^{\prime}, v^{\prime}\right]$ as defined in (4.5) which, in turn, is given in terms of the integrals (4.6,4.7). First we calculate in RS ansatz the $n \rightarrow 0$ limit of $D[u, v ; q,\{\chi\}]$ (4.6), using (4.14), and simplify the result with the saddle-point equation (4.17):

$$
\begin{aligned}
& \lim _{n \rightarrow 0} \mathcal{D}[u, v ; q,\{\chi\}]= \int D z D y e^{-i v y} \frac{\int d x e^{-\frac{x^{2}}{2 Q(1-q)}+x[A y+B z]+\frac{1}{\alpha} \chi[x, y]-i u x}}{\int d x e^{-\frac{x^{2}}{2 Q(1-q)}+x[A y+B z]+\frac{1}{\alpha} \chi[x, y]}} \\
&=\int d x d y P[x, y] e^{-i v y-i u x}
\end{aligned}
$$

Next we work out $F_{\lambda}^{\alpha}[u, v]$ (4.7) in RS ansatz, using (4.14), with $\lambda \in\{1,2\}$ :

$$
\begin{aligned}
& \lim _{n \rightarrow 0} \mathcal{F}_{\lambda}^{\alpha}[u, v]=i \lim _{n \rightarrow 0} \\
& \int D y D z e^{-i v y} \int d \boldsymbol{x} e^{\sum_{\beta}\left[-\frac{1}{2} \frac{x_{\beta}^{2}}{1-q}+[z \sqrt{d}-\gamma y] x_{\beta}+\frac{1}{\alpha} \chi\left[\sqrt{Q} x_{\beta}, y\right]\right]-i u x_{1} \sqrt{Q}} \partial_{\lambda} \chi\left[\sqrt{Q} x_{\alpha}, y\right]
\end{aligned}
$$

Replica permutation symmetries allow us to simplify this expression:

$$
\lim _{n \rightarrow 0} \mathcal{F}_{\lambda}^{\alpha}[u, v]=\delta_{\alpha 1} F_{\lambda}^{1}[u, v]+\left(1-\delta_{\alpha 1}\right) F_{\lambda}^{2}[u, v]
$$


with

$$
F_{\lambda}^{1}[u, v]=i \int d x d y P[x, y] e^{-i v y-i u x} \partial_{\lambda} \chi[x, y]
$$

and

$$
\begin{aligned}
& F_{\lambda}^{2}[u, v]=i \int D y D z e^{-i v y} \\
& \frac{\left[\int d x e^{-\frac{x^{2}}{2 Q(1-q)}+x[A y+B z]+\frac{1}{\alpha} \chi[x, y]} \partial_{\lambda} \chi[x, y]\right]\left[\int d x e^{-\frac{x^{2}}{2 Q(1-q)}+x[A y+B z]+\frac{1}{\alpha} \chi[x, y]-i u x}\right]}{\left[\int d x e^{-\frac{x^{2}}{2 Q(1-q)}+x[A y+B z]+\frac{1}{\alpha} \chi[x, y]}\right]^{2}}
\end{aligned}
$$

We can now proceed with the calculation of (4.5), whose building blocks are

$$
\begin{aligned}
\alpha^{-1} \mathcal{F}_{1}^{\alpha}[u, v]+u \delta_{\alpha 1} \mathcal{D}[u, v] & =\delta_{\alpha 1} G_{1}[u, v]+\left(1-\delta_{\alpha 1}\right) \tilde{G}_{1,2}[u, v] \\
\alpha^{-1} \mathcal{F}_{2}^{\alpha}[u, v]+v \delta_{\alpha 1} \mathcal{D}[u, v] & =\delta_{\alpha 1} G_{2}[u, v]+\left(1-\delta_{\alpha 1}\right) \tilde{G}_{2}[u, v]
\end{aligned}
$$

with

$$
\begin{array}{rlrl}
G_{1}[u, v] & =\alpha^{-1} \mathcal{F}_{1,2}^{1}[u, v]+u \mathcal{D}[u, v] & & \tilde{G}_{1}[u, v]=\alpha^{-1} \mathcal{F}_{1}^{2}[u, v] \\
G_{2}[u, v]=\alpha^{-1} \mathcal{F}_{2}^{1}[u, v]+v \mathcal{D}[u, v] & \tilde{G}_{2}[u, v]=\alpha^{-1} \mathcal{F}_{2}^{2}[u, v]
\end{array}
$$

and their Fourier transforms:

$$
\begin{array}{ll}
\hat{G}_{1}[\hat{u}, \hat{v}]=\int \frac{d u d v}{(2 \pi)^{2}} e^{i u \hat{u}+i v \hat{v}} G_{1}[u, v] & \bar{G}_{1}[\hat{u}, \hat{v}]=\int \frac{d u d v}{(2 \pi)^{2}} e^{i u \hat{u}+i v \hat{v}} \tilde{G}_{1}[u, v] \\
\hat{G}_{2}[\hat{u}, \hat{v}]=\int \frac{d u d v}{(2 \pi)^{2}} e^{i u \hat{u}+i v \hat{v}} G_{2}[u, v] & \bar{G}_{2}[\hat{u}, \hat{v}]=\int \frac{d u d v}{(2 \pi)^{2}} e^{i u \hat{u}+i v \hat{v}} \tilde{G}_{2}[u, v]
\end{array}
$$

With these short-hands we obtain a compact expression for (4.5), and can subsequently write our expression (4.9) for the Green's function $\mathcal{A}\left[x, y ; x^{\prime}, y^{\prime}\right]$ as

$$
\begin{aligned}
\mathcal{A}\left[x, y ; x^{\prime}, y^{\prime}\right] & =-Q(1-q)\left[\hat{G}_{1}[x, y] \hat{G}_{1}\left[x^{\prime}, y^{\prime}\right]-\bar{G}_{1}[x, y] \bar{G}_{1}\left[x^{\prime}, y^{\prime}\right]\right] \\
& -Q q\left[\hat{G}_{1}[x, y]-\bar{G}_{1}[x, y]\right]\left[\hat{G}_{1}\left[x^{\prime}, y^{\prime}\right]-\bar{G}_{1}\left[x^{\prime}, y^{\prime}\right]\right] \\
& -R\left[\hat{G}_{1}[x, y]-\bar{G}_{1}[x, y]\right]\left[\hat{G}_{2}\left[x^{\prime}, y^{\prime}\right]-\bar{G}_{2}\left[x^{\prime}, y^{\prime}\right]\right] \\
& -R\left[\hat{G}_{1}\left[x^{\prime}, y^{\prime}\right]-\bar{G}_{1}\left[x^{\prime}, y^{\prime}\right]\right]\left[\hat{G}_{2}[x, y]-\bar{G}_{2}[x, y]\right] \\
& -\left[\hat{G}_{2}[x, y]-\bar{G}_{2}[x, y]\right]\left[\hat{G}_{2}\left[x^{\prime}, y^{\prime}\right]-\bar{G}_{2}\left[x^{\prime}, y^{\prime}\right]\right]
\end{aligned}
$$

Finally, working out the four relevant Fourier transforms, using (4.19,4.21,4.22), gives:

$$
\begin{aligned}
& \hat{G}_{1}[x, y]=i\left[\frac{1}{\alpha} P[x, y] \frac{\partial}{\partial x} \chi[x, y]-\frac{\partial}{\partial x} P[x, y]\right] \\
& \hat{G}_{2}[x, y]=i\left[\frac{1}{\alpha} P[x, y] \frac{\partial}{\partial y} \chi[x, y]-\frac{\partial}{\partial y} P[x, y]\right]
\end{aligned}
$$




$$
\begin{aligned}
& \bar{G}_{1}[x, y]=\frac{i}{\alpha} \frac{e^{-\frac{1}{2} y^{2}}}{\sqrt{2 \pi}} \int D z \\
& \frac{\left[\int d x^{\prime} e^{-\frac{x^{\prime 2}}{2 Q(1-q)}+x^{\prime}[A y+B z]+\frac{1}{\alpha} \chi\left[x^{\prime}, y\right]} \partial_{1} \chi\left[x^{\prime}, y\right]\right] e^{-\frac{x^{2}}{2 Q(1-q)}+x[A y+B z]+\frac{1}{\alpha} \chi[x, y]}}{\left[\int d x^{\prime} e^{\left.-\frac{x^{\prime 2}}{2 Q(1-q)}+x^{\prime}[A y+B z]+\frac{1}{\alpha} \chi\left[x^{\prime}, y\right]\right]^{2}}\right.} \\
& \bar{G}_{2}[x, y]=\frac{i}{\alpha} \frac{e^{-\frac{1}{2} y^{2}}}{\sqrt{2 \pi}} \int D z \\
& {\left[\int d x^{\prime} e^{-\frac{x^{\prime 2}}{2 Q(1-q)}+x^{\prime}[A y+B z]+\frac{1}{\alpha} \chi\left[x^{\prime}, y\right]} \partial_{2} \chi\left[x^{\prime}, y\right]\right] e^{-\frac{x^{2}}{2 Q(1-q)}+x[A y+B z]+\frac{1}{\alpha} \chi[x, y]}} \\
& {\left[\int d x^{\prime} e^{\left.-\frac{x^{\prime 2}}{2 Q(1-q)}+x^{\prime}[A y+B z]+\frac{1}{\alpha} \chi\left[x^{\prime}, y\right]\right]^{2}}\right.}
\end{aligned}
$$

\subsection{Summary}

At this stage it is advantageous to summarize the theory and choose the most transparent representation of our equations. We first replace the function $\chi[x, y]$ by the effective measure $M[x, y]$ :

$$
M[x, y]=e^{-\frac{x^{2}}{2 Q(1-q)}+A x y+\frac{1}{\alpha} \chi\left[x^{\prime}, y\right]}
$$

We introduce a compact notation for the various averages we encounter:

$$
\langle f[x, y, z]\rangle_{\star}=\frac{\int d x M[x, y] e^{B x z} f[x, y, z]}{\int d x M[x, y] e^{B x z}} \quad\langle f[y, z]\rangle=\int D y D z f[y, z]
$$

with the short-hand

$$
B=\frac{\sqrt{q Q-R^{2}}}{Q(1-q)}
$$

From equation (4.17) we deduce that the function $P[x, y]$ always obeys

$$
P[x, y]=P[x \mid y] P[y] \quad P[y]=(2 \pi)^{-\frac{1}{2}} e^{-\frac{1}{2} y^{2}}
$$

This enables us to write our results in terms of $P[x \mid y]$ rather than $P[x, y]$. We also introduce the transformed Green's function $\tilde{\mathcal{A}}$ via $\mathcal{A}\left[x, y ; x^{\prime}, y^{\prime}\right]=$ $P[x, y] \tilde{\mathcal{A}}\left[x, y ; x^{\prime}, y^{\prime}\right] P\left[x^{\prime}, y^{\prime}\right]$. In combination these simplifications allow us to summarize our theory as follows. The macroscopic dynamic equations are:

$$
\begin{gathered}
\frac{d}{d t} Q=2 \eta \int d x D y P[x \mid y] x \mathcal{G}[x, y]+L \quad \frac{d}{d t} R=\eta \int d x D y P[x \mid y] y \mathcal{G}[x, y] \\
\frac{\partial}{\partial t} P[x \mid y]=-\eta \frac{\partial}{\partial x}\left\{P[x \mid y]\left[\frac{1}{\alpha} \mathcal{G}[x, y]+\int d x^{\prime} D y^{\prime} P\left[x^{\prime} \mid y^{\prime}\right] \mathcal{G}\left[x^{\prime}, y^{\prime}\right] \tilde{\mathcal{A}}\left[x, y ; x^{\prime}, y^{\prime}\right]\right]\right\} \\
+\frac{1}{2} L \frac{\partial^{2}}{\partial x^{2}} P[x \mid y]
\end{gathered}
$$


with

$$
\text { batch : } L=0 \quad \text { on-line : } L=\eta^{2} \int d x D y P[x \mid y] \mathcal{G}^{2}[x, y]
$$

The solution of (4.26,4.27) subsequently generates the training- and generalization errors (2.5,2.6) at any time:

$$
E_{\mathrm{t}}=\int d x D y P[x \mid y] \theta[-x y] \quad E_{\mathrm{g}}=\frac{1}{\pi} \arccos [R / \sqrt{Q}]
$$

In order to determine the Green's function $\tilde{\mathcal{A}}\left[x, y ; x^{\prime}, y^{\prime}\right]$ in equation (4.27) one first has to calculate the auxiliary order parameters $q$ and $\{M[x, y]\}$ by solving the following two saddle point equations:

$$
\begin{gathered}
\text { for all } X, y: \quad P[X \mid y]=\int D z\langle\delta[X-x]\rangle_{\star} \\
\int d x D y P[x \mid y]\left(x^{2}-2 R x y\right)-q Q\left(\frac{1}{\alpha}-1\right)+\frac{R^{2}}{\alpha}=\left[2 \sqrt{q Q-R^{2}}+\frac{1}{B}\right] \int D y D z z\langle x\rangle_{\star}
\end{gathered}
$$

The Green's function in equation (4.27) is then given by

$$
\begin{array}{r}
\tilde{\mathcal{A}}\left[x, y ; x^{\prime}, y^{\prime}\right]=Q(1-q)\left[J_{1}[x, y] J_{1}\left[x^{\prime}, y^{\prime}\right]-\tilde{J}_{1}[x, y] \tilde{J}_{1}\left[x^{\prime}, y^{\prime}\right]\right] \\
+Q q\left[J_{1}[x, y]-\tilde{J}_{1}[x, y]\right]\left[J_{1}\left[x^{\prime}, y^{\prime}\right]-\tilde{J}_{1}\left[x^{\prime}, y^{\prime}\right]\right]+J_{2}[x, y] J_{2}\left[x^{\prime}, y^{\prime}\right] \\
+R\left[J_{1}[x, y]-\tilde{J}_{1}[x, y]\right] J_{2}\left[x^{\prime}, y^{\prime}\right]+R\left[J_{1}\left[x^{\prime}, y^{\prime}\right]-\tilde{J}_{1}\left[x^{\prime}, y^{\prime}\right]\right] J_{2}[x, y]
\end{array}
$$

with the functions

$$
\begin{aligned}
& J_{1}[X, Y]=\frac{\partial}{\partial X} \log \frac{M[X, Y]}{P[X \mid Y]}+\frac{X-R Y}{Q(1-q)} \\
& \tilde{J}_{1}[X, Y]=P[X \mid Y]^{-1} \int D z\left\langle\frac{\partial}{\partial x} \log M[x, Y]+\frac{x-R Y}{Q(1-q)}\right\rangle_{\star}\langle\delta[X-x]\rangle_{\star} \\
& J_{2}[X, Y]=\frac{\partial}{\partial Y} \log \frac{M[X, Y]}{P[X \mid Y]}-\frac{R X}{Q(1-q)}+Y \\
& -P[X \mid Y]^{-1} \int D z\left\langle\frac{\partial}{\partial Y} \log M[x, Y]-\frac{R x}{Q(1-q)}\right\rangle_{\star}\langle\delta[X-x]\rangle_{\star}
\end{aligned}
$$

We finally work out a simple inequality to determine for which regime of $q$ values one should inspect the saddle-point equations. From (4.12) it follows that $q=\left\langle\frac{1}{N} \sum_{i}\left\langle\sigma_{i}\right\rangle^{2}\right\rangle \mathbf{\Xi}$, giving

$$
0 \leq\left\langle\frac{1}{N} \sum_{i}\left[\left\langle\sigma_{i}\right\rangle-\tau_{i}\left[\frac{1}{N} \sum_{j} \tau_{j}\left\langle\sigma_{j}\right\rangle\right]\right]^{2}\right\rangle_{\Xi}=q-R^{2} / Q
$$




\section{Applications and Tests of the Theory}

\subsection{The Limit $\alpha \rightarrow \infty$}

The very least we should require of our theory is that it reduces to the simple $(Q, R)$ formalism of infinite training sets in the limit $\alpha \rightarrow \infty$. This indeed happens. For $\alpha \rightarrow \infty$ our macroscopic equations (4.26,4.27) reduce to

$\frac{d}{d t} Q=2 \eta \int d x D y P[x \mid y] x \mathcal{G}[x ; y]+L \quad \frac{d}{d t} R=\eta \int d x D y P[x \mid y] y \mathcal{G}[x ; y]$

$\frac{\partial}{\partial t} P[x \mid y]=-\eta \frac{\partial}{\partial x}\left\{P[x \mid y] \int d x^{\prime} D y^{\prime} P\left[x^{\prime} \mid y^{\prime}\right] \mathcal{G}\left[x^{\prime}, y^{\prime}\right] \tilde{\mathcal{A}}\left[x, y ; x^{\prime}, y^{\prime}\right]\right\}+\frac{L}{2} \frac{\partial^{2}}{\partial x^{2}} P[x \mid y]$

with the Green's function $\tilde{\mathcal{A}}\left[x, y ; x^{\prime}, y^{\prime}\right]$ (4.32), and with $L$ as given in (4.28). The saddle-point equations from which to solve $\{M[x, y]\}$ and $q$ are:

$$
\begin{gathered}
P[X \mid y]=\int D z\langle\delta[X-x]\rangle_{\star} \\
q Q+\int d x D y P[x \mid y]\left(x^{2}-2 R x y\right)=\left[2 \sqrt{q Q-R^{2}}+B^{-1}\right] \int D y D z z\langle x\rangle_{\star}
\end{gathered}
$$

with the convention (4.24), and with the short-hand $B=\sqrt{q Q-R^{2}} / Q(1-q)$. We now make the following ansatz:

$$
P[x \mid y]=\left[2 \pi\left(Q-R^{2}\right)\right]^{-\frac{1}{2}} e^{-\frac{1}{2}[x-R y]^{2} /\left(Q-R^{2}\right)}
$$

and find that the two saddle-point equations are simultaneously solved by

$$
M[x, y]=[2 \pi Q(1-q)]^{-\frac{1}{2}} e^{-\frac{1}{2}[x-R y]^{2} / Q(1-q)}
$$

(we will return to the question of uniqueness later). The values of $Q$ and $R$ at any time are fully specified by (5.1); what remains is to verify the validity of (5.2). Given the measure (5.4) one can calculate the three functions $J_{1}[x, y]$, $J_{1}[x, y]$ and $J_{2}[x, y]$, and thus the Green's function (4.32), explicitly:

$$
\begin{gathered}
J_{1}[x, y]=\frac{x-R y}{Q-R^{2}} \quad \tilde{J}_{1}[x, y]=0 \quad J_{2}[x, y]=\frac{Q y-R x}{Q-R^{2}} \\
\tilde{\mathcal{A}}\left[x, y ; x^{\prime}, y^{\prime}\right]=\frac{(x-R y) x^{\prime}+(Q y-R x) y^{\prime}}{Q-R^{2}}
\end{gathered}
$$

This, in combination with the equations (5.1) for $Q$ and $R$, leads to an explicit expression for the diffusion equation (5.2):

$\frac{\partial}{\partial t} P[x \mid y]=\frac{\partial}{\partial x}\left\{\frac{L}{2} \frac{\partial}{\partial x} P[x \mid y]-\frac{P[x \mid y]}{Q-R^{2}}\left[\frac{1}{2}(x-R y)\left[\frac{d Q}{d t}-L\right]+(Q y-R x) \frac{d R}{d t}\right]\right\}$

Insertion of our ansatz (5.3) into both sides of this equation, followed by some rearranging of terms, shows that it is indeed satisfied. This confirms that from our general theory we indeed recover for $\alpha \rightarrow \infty$ the standard theory for infinite training sets, i.e. the closed set (5.1, 5.3), as claimed. 


\subsection{Locally Gaussian Solutions}

As soon as $\alpha$ is finite the diffusion equation (4.27) will no longer have Gaussian solutions. However, we will show in this section that for a specific simple class of learning rules one can find solutions described by a conditional distribution $P[x \mid y]$ which for each $y$ is Gaussian in $x$, but with moments which are nontrivial functions of $y$ (such that the full distribution $P[x, y]$ is not Gaussian):

$$
\mathcal{G}[x, y]=\mathcal{G}_{0}[y]+x \mathcal{G}_{1}[y]: \quad P[x \mid y]=\frac{e^{-\frac{1}{2}[x-\bar{x}(y)]^{2} / \Delta^{2}(y)}}{\Delta(y) \sqrt{2 \pi}}
$$

We choose the distribution $P[x \mid y]$ in (5.6) as our ansatz. Insertion into the saddle-point equation (4.30) and integration over $z$ shows that (4.30) is solved by the following measure (the issue of uniqueness will be discussed later):

$$
M[x, y]=\frac{e^{-\frac{1}{2}[x-\bar{x}(y)]^{2} / \sigma^{2}(y)}}{\sigma(y) \sqrt{2 \pi}}
$$

with the usual short-hand $B=\sqrt{q Q-R^{2}} / Q(1-q)$, and with

$$
\sigma^{2}(y)=\frac{1}{2 B^{2}}\left[\sqrt{1+4 B^{2} \Delta^{2}(y)}-1\right]
$$

The simple form of (5.7) allows us to proceed analytically, using identities such as

$$
\langle x\rangle_{\star}=\bar{x}(y)+B z \sigma^{2}(y) \quad\left\langle[x-\bar{x}(y)]^{2}\right\rangle_{\star}=\sigma^{2}(y)+B^{2} z^{2} \sigma^{4}(y)
$$

The remaining saddle point equation (4.31) for $q$ can be simplified to

$$
\int D y\left[\Delta^{2}(y)+\bar{x}^{2}(y)-2 R y \bar{x}(y)\right]-q Q\left(\frac{1}{\alpha}-1\right)+\frac{R^{2}}{\alpha}=\left[\frac{2\left(q Q-R^{2}\right)}{Q(1-q)}+1\right] \int D y \sigma^{2}(y)
$$

Next we have to show that our ansatz (5.6), in combination with (5.8,5.9), solves the dynamic equations (4.26,4.27). In order to do so we first calculate the building blocks of the Green's function $\tilde{A}\left[x, y ; x^{\prime}, y^{\prime}\right]$, giving (after a modest amount of bookkeeping):

$J_{1}[x, y]=x V_{1}(y)+V_{2}(y) \quad \tilde{J}_{1}[x, y]=x \tilde{V}_{1}(y)+\tilde{V}_{2}(y) \quad J_{2}[x, y]=x W_{1}(y)+W_{2}(y)$

with the six functions

$$
\begin{gathered}
V_{1}(y)=\frac{\sigma^{2}(y) \Delta^{2}(y)+Q(1-q)\left[\sigma^{2}(y)-\Delta^{2}(y)\right]}{Q(1-q) \sigma^{2}(y) \Delta^{2}(y)} \\
V_{2}(y)=\frac{\bar{x}(y) Q(1-q)\left[\Delta^{2}(y)-\sigma^{2}(y)\right]-R y \sigma^{2}(y) \Delta^{2}(y)}{Q(1-q) \sigma^{2}(y) \Delta^{2}(y)}
\end{gathered}
$$




$$
\begin{gathered}
\tilde{V}_{1}(y)=\frac{\left[\sigma^{2}(y)-\Delta^{2}(y)\right]\left[Q(1-q)-\sigma^{2}(y)\right]}{Q(1-q) \sigma^{2}(y) \Delta^{2}(y)} \\
\tilde{V}_{2}(y)=\frac{\bar{x}(y)\left[\Delta^{2}(y)-\sigma^{2}(y)\right]\left[Q(1-q)-\sigma^{2}(y)\right]+(\bar{x}(y)-R y) \Delta^{2}(y) \sigma^{2}(y)}{Q(1-q) \sigma^{2}(y) \Delta^{2}(y)} \\
W_{1}(y)=-\frac{R \sigma^{4}(y)}{Q(1-q) \sigma^{2}(y) \Delta^{2}(y)} \\
W_{2}(y)=\frac{\left.y \sigma^{2}(y) \Delta^{2}(y) Q(1-q)+R \bar{x}(y) \sigma^{4}(y)\right]}{Q(1-q) \sigma^{2}(y) \Delta^{2}(y)}
\end{gathered}
$$

Insertion of these expressions into the Green's function (4.32) subsequently gives the simple expression

$$
\tilde{\mathcal{A}}\left[x, y ; x^{\prime}, y^{\prime}\right]=x x^{\prime} U_{1}\left(y, y^{\prime}\right)+x U_{2}\left(y, y^{\prime}\right)+x^{\prime} U_{2}\left(y^{\prime}, y\right)+U_{3}\left(y, y^{\prime}\right)
$$

with the three kernels

$$
\begin{aligned}
U_{1}\left(y, y^{\prime}\right) & =W_{1}(y) W_{1}\left(y^{\prime}\right) \\
+Q(1-q) & {\left[V_{1}(y) V_{1}\left(y^{\prime}\right)-\tilde{V}_{1}(y) \tilde{V}_{1}\left(y^{\prime}\right)\right]+Q q\left[V_{1}(y)-\tilde{V}_{1}(y)\right]\left[V_{1}\left(y^{\prime}\right)-\tilde{V}_{1}\left(y^{\prime}\right)\right] } \\
& +R\left[V_{1}(y)-\tilde{V}_{1}(y)\right] W_{1}\left(y^{\prime}\right)+R W_{1}(y)\left[V_{1}\left(y^{\prime}\right)-\tilde{V}_{1}\left(y^{\prime}\right)\right] \\
U_{2}\left(y, y^{\prime}\right) & =W_{1}(y) W_{2}\left(y^{\prime}\right) \\
+Q(1-q) & {\left[V_{1}(y) V_{2}\left(y^{\prime}\right)-\tilde{V}_{1}(y) \tilde{V}_{2}\left(y^{\prime}\right)\right]+Q q\left[V_{1}(y)-\tilde{V}_{1}(y)\right]\left[V_{2}\left(y^{\prime}\right)-\tilde{V}_{2}\left(y^{\prime}\right)\right] } \\
& +R\left[V_{1}(y)-\tilde{V}_{1}(y)\right] W_{2}\left(y^{\prime}\right)+R W_{1}(y)\left[V_{2}\left(y^{\prime}\right)-\tilde{V}_{2}\left(y^{\prime}\right)\right] \\
U_{3}\left(y, y^{\prime}\right) & =W_{2}(y) W_{2}\left(y^{\prime}\right) \\
+Q(1-q) & {\left[V_{2}(y) V_{2}\left(y^{\prime}\right)-\tilde{V}_{2}(y) \tilde{V}_{2}\left(y^{\prime}\right)\right]+Q q\left[V_{2}(y)-\tilde{V}_{2}(y)\right]\left[V_{2}\left(y^{\prime}\right)-\tilde{V}_{2}\left(y^{\prime}\right)\right] } \\
& +R\left[V_{2}(y)-\tilde{V}_{2}(y)\right] W_{2}\left(y^{\prime}\right)+R W_{2}(y)\left[V_{2}\left(y^{\prime}\right)-\tilde{V}_{2}\left(y^{\prime}\right)\right]
\end{aligned}
$$

Insertion of the above expression (5.10) for the Green's function into the right-hand side of our diffusion equation (4.27) gives, in turn:

$$
\begin{aligned}
\frac{\Delta^{2}(y)}{P[x \mid y]} \text { RHS } & =-\frac{L}{2}\left[1-\frac{[x-\bar{x}(y)]^{2}}{\Delta^{2}(y)}\right]+\eta[x-\bar{x}(y)]\left\{\frac{1}{\alpha} \mathcal{G}[x, y]\right. \\
+ & {[x-\bar{x}(y)] \int d x^{\prime} D y^{\prime} P\left[x^{\prime} \mid y^{\prime}\right] \mathcal{G}\left[x^{\prime}, y^{\prime}\right]\left[x^{\prime} U_{1}\left(y, y^{\prime}\right)+U_{2}\left(y, y^{\prime}\right)\right] } \\
+ & \bar{x}(y) \int d x^{\prime} D y^{\prime} P\left[x^{\prime} \mid y^{\prime}\right] \mathcal{G}\left[x^{\prime}, y^{\prime}\right]\left[x^{\prime} U_{1}\left(y, y^{\prime}\right)+U_{2}\left(y, y^{\prime}\right)\right] \\
+ & \left.\int d x^{\prime} D y^{\prime} P\left[x^{\prime} \mid y^{\prime}\right] \mathcal{G}\left[x^{\prime}, y^{\prime}\right]\left[x^{\prime} U_{2}\left(y^{\prime}, y\right)+U_{3}\left(y, y^{\prime}\right)\right]\right\} \\
-\eta \Delta^{2}(y) & \left\{\frac{1}{\alpha} \frac{\partial}{\partial x} \mathcal{G}[x, y]+\int d x^{\prime} D y^{\prime} P\left[x^{\prime} \mid y^{\prime}\right] \mathcal{G}\left[x^{\prime}, y^{\prime}\right]\left[x^{\prime} U_{1}\left(y, y^{\prime}\right)+U_{2}\left(y, y^{\prime}\right)\right]\right\}
\end{aligned}
$$


For the left-hand side of the diffusion equation for $P[x \mid y]$ we get

$$
\frac{\Delta^{2}(y)}{P[x \mid y]} \text { LHS }=[x-\bar{x}(y)] \frac{d}{d t} \bar{x}(y)+\left[\frac{[x-\bar{x}(y)]^{2}}{\Delta(y)}-\Delta(y)\right] \frac{d}{d t} \Delta(y)
$$

Since LHS is a second-order polynomial in $x$, we have to restrict ourselves to functions $\mathcal{G}[x, y]$ which are first-order polynomials in $x$, hence the definition (5.6). We equate the three monomials of $x-\bar{x}(y)$ and find for self-consistent solutions of the locally Gaussian type (5.6) the following conditions:

$$
\begin{aligned}
{[x-\bar{x}(y)]^{2}: } & \frac{d}{d t} \Delta(y)=\frac{L}{2 \Delta(y)}+\eta \Delta(y)\left\{\alpha^{-1} \mathcal{G}_{1}(y)\right. \\
+ & \left.\int d x^{\prime} D y^{\prime} P\left[x^{\prime} \mid y^{\prime}\right] \mathcal{G}\left[x^{\prime}, y^{\prime}\right]\left[x^{\prime} U_{1}\left(y, y^{\prime}\right)+U_{2}\left(y, y^{\prime}\right)\right]\right\} \\
{[x-\bar{x}(y)]: \quad \frac{d}{d t} \bar{x}(y) } & =\eta\left\{\alpha^{-1}\left[\mathcal{G}_{0}(y)+\bar{x}(y) \mathcal{G}_{1}(y)\right]\right. \\
+ & \bar{x}(y) \int d x^{\prime} D y^{\prime} P\left[x^{\prime} \mid y^{\prime}\right] \mathcal{G}\left[x^{\prime}, y^{\prime}\right]\left[x^{\prime} U_{1}\left(y, y^{\prime}\right)+U_{2}\left(y, y^{\prime}\right)\right] \\
& \left.+\int d x^{\prime} D y^{\prime} P\left[x^{\prime} \mid y^{\prime}\right] \mathcal{G}\left[x^{\prime}, y^{\prime}\right]\left[x^{\prime} U_{2}\left(y^{\prime}, y\right)+U_{3}\left(y, y^{\prime}\right)\right]\right\} \\
{[x-\bar{x}(y)]^{0}: \quad \frac{L}{d t} \Delta(y) } & =\frac{L}{2 \Delta(y)}+\eta \Delta(y)\left\{\alpha^{-1} \mathcal{G}_{1}(y)\right. \\
& \left.+\int d x^{\prime} D y^{\prime} P\left[x^{\prime} \mid y^{\prime}\right] \mathcal{G}\left[x^{\prime}, y^{\prime}\right]\left[x^{\prime} U_{1}\left(y, y^{\prime}\right)+U_{2}\left(y, y^{\prime}\right)\right]\right\}
\end{aligned}
$$

This is an important self-consistency test, since we have three equations for only two functions $(\bar{x}(y)$ and $\Delta(y))$. Two of the three equations are found to be identical. In the remaining two equations we insert the form of the learning rules (5.6) and perform the $x$-integrations, giving

$$
\begin{aligned}
\frac{d}{d t} \Delta(y) & =\frac{L}{2 \Delta(y)}+\eta \Delta(y)\left\{\frac{\mathcal{G}_{1}(y)}{\alpha}+\int D y^{\prime} \mathcal{G}_{0}\left(y^{\prime}\right)\left[\bar{x}\left(y^{\prime}\right) U_{1}\left(y, y^{\prime}\right)+U_{2}\left(y, y^{\prime}\right)\right]\right. \\
+ & \left.\int D y^{\prime} \mathcal{G}_{1}\left(y^{\prime}\right)\left[\left[\bar{x}^{2}\left(y^{\prime}\right)+\Delta^{2}\left(y^{\prime}\right)\right] U_{1}\left(y, y^{\prime}\right)+\bar{x}\left(y^{\prime}\right) U_{2}\left(y, y^{\prime}\right)\right]\right\} \\
\frac{d}{d t} \bar{x}(y) & =\eta\left\{\frac{1}{\alpha}\left[\mathcal{G}_{0}(y)+\bar{x}(y) \mathcal{G}_{1}(y)\right]+\bar{x}(y) \int D y^{\prime} \mathcal{G}_{0}\left(y^{\prime}\right)\left[\bar{x}\left(y^{\prime}\right) U_{1}\left(y, y^{\prime}\right)+U_{2}\left(y, y^{\prime}\right)\right]\right. \\
& +\bar{x}(y) \int D y^{\prime} \mathcal{G}_{1}\left(y^{\prime}\right)\left[\left[\bar{x}^{2}\left(y^{\prime}\right)+\Delta^{2}\left(y^{\prime}\right)\right] U_{1}\left(y, y^{\prime}\right)+\bar{x}\left(y^{\prime}\right) U_{2}\left(y, y^{\prime}\right)\right] \\
& +\int D y^{\prime} \mathcal{G}_{0}\left(y^{\prime}\right)\left[\bar{x}\left(y^{\prime}\right) U_{2}\left(y^{\prime}, y\right)+U_{3}\left(y, y^{\prime}\right)\right] \\
+ & \left.\int D y^{\prime} \mathcal{G}_{1}\left(y^{\prime}\right)\left[\left[\bar{x}^{2}\left(y^{\prime}\right)+\Delta^{2}\left(y^{\prime}\right)\right] U_{2}\left(y^{\prime}, y\right)+\bar{x}\left(y^{\prime}\right) U_{3}\left(y, y^{\prime}\right)\right]\right\}
\end{aligned}
$$

Our result is a solution in the form of coupled equations 4.26,5.6, 5.9, 5.14, 5.15), without a functional saddle-point problem and without a diffusion equation. 


\subsection{Explicit Example: Hebbian Learning}

The simplest non-trivial member of the family (5.6) of learning rules with locally Gaussian field distributions is the Hebb rule: $\mathcal{G}[x, y]=\operatorname{sgn}[y]$ (i.e. $\mathcal{G}_{0}(y)=\operatorname{sgn}(y)$ and $\left.\mathcal{G}_{1}(y)=0\right)$. This example we will work out in full, as an explicit illustration and in order to have a precise test (since only for this rule one can calculate the macroscopic observables exactly and directly from the microscopic laws). The remaining integrals in our equations 4.26,5.6, $5.9,5.14,5.15)$ can be carried out explicitly. We find (for initial states $\boldsymbol{J}(0)$ which are not correlated with the questions in the training set):

$$
R=R_{0}+\eta t \sqrt{\frac{2}{\pi}} \quad Q=Q_{0}+\eta t\left[\kappa \eta+2 R_{0} \sqrt{\frac{2}{\pi}}\right]+\eta^{2} t^{2}\left[\frac{2}{\pi}+\frac{1}{\alpha}\right]
$$

(with $\kappa=1$ for on-line learning and $\kappa=0$ for batch learning), and

$$
\bar{x}(y)=R y+\alpha^{-1} \eta t \operatorname{sgn}(y)
$$

To find the width $\Delta$ of $P[x \mid y]$ (which is found to remain independent of $y$, if so at $t=0)$, we have to solve the coupled equations (5.9,5.14), which for the Hebb rule reduce to

$$
\begin{gathered}
\frac{1}{\eta} \frac{d}{d t} \Delta^{2}=\kappa \eta+\frac{\eta t}{\alpha} \frac{Q(1-q)}{q Q-R^{2}}\left[\sqrt{1+\frac{4\left(q Q-R^{2}\right) \Delta^{2}}{Q^{2}(1-q)^{2}}}-1\right] \\
\left(R^{2}-q Q\right)\left(\frac{1}{\alpha}-1\right)+\Delta^{2}+\frac{\eta^{2} t^{2}}{\alpha^{2}}=Q(1-q)\left[1+\frac{Q(1-q)}{2\left(q Q-R^{2}\right)}\right]\left[\sqrt{1+\frac{4\left(q Q-R^{2}\right) \Delta^{2}}{Q^{2}(1-q)^{2}}}-1\right]
\end{gathered}
$$

The solution of these equations is given by

$$
\Delta^{2}=Q-R^{2} \quad q=Q^{-1}\left[R^{2}+\alpha^{-1} \eta^{2} t^{2}\right]
$$

This solution is unique (for a proof see Coolen and Saad, 1998). Thus:

$$
P[x \mid y]=\frac{e^{-\frac{1}{2}\left[x-R y-\alpha^{-1} \eta t \operatorname{sgn}(y)\right]^{2} /\left(Q-R^{2}\right)}}{\sqrt{2 \pi\left(Q-R^{2}\right)}}
$$

We can now also calculate both errors as a functions of time:

$$
E_{\mathrm{g}}=\frac{1}{\pi} \arccos \left[\frac{R}{\sqrt{Q}}\right] \quad E_{\mathrm{t}}=\frac{1}{2}-\frac{1}{2} \int D y \operatorname{erf}\left[\frac{|y| R+\eta t / \alpha}{\sqrt{2\left(Q-R^{2}\right)}}\right]
$$

Asymptotically one finds $\lim _{t \rightarrow \infty} q=1$, and

$$
\lim _{t \rightarrow \infty} E_{\mathrm{g}}=\frac{1}{\pi} \arccos \left[\frac{\sqrt{2 \alpha}}{\sqrt{2 \alpha+\pi}}\right] \quad \lim _{t \rightarrow \infty} E_{\mathrm{t}}=\frac{1}{2}-\frac{1}{2} \int D y \operatorname{erf}\left[|y| \sqrt{\frac{\alpha}{\pi}}+\frac{1}{\sqrt{2 \alpha}}\right]
$$




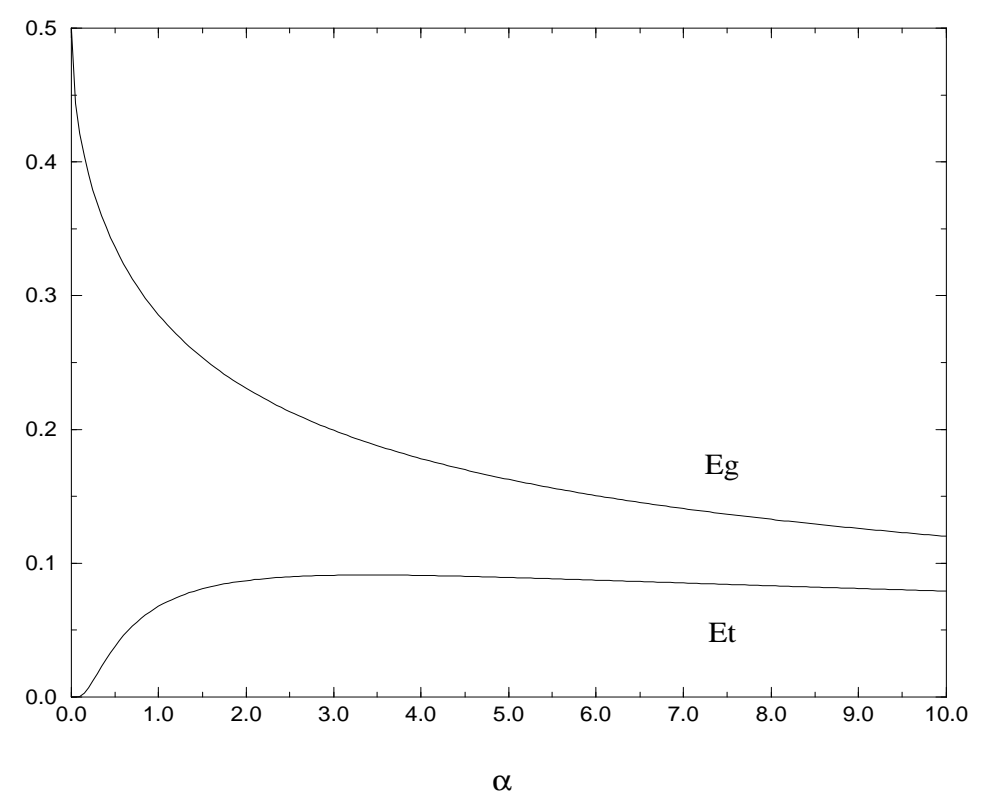

Figure 4: Fig. 4: Asymptotic values of the two errors, i.e. $\lim _{t \rightarrow \infty} E_{\mathrm{g}}$ and $\lim _{t \rightarrow \infty} E_{\mathrm{t}}$, for Hebbian learning (both on-line and batch), as functions of the relative size $\alpha=p / N$ of the training set.

Both asymptotic errors are independent of $\kappa$, i.e. on whether batch or on-line learning is used. These results are depicted in Fig. 4.

A final object to be calculated is the student field distribution $P(x)$ itself, via $P(x)=\int d y P[x, y]=\int D y P[x \mid y]$. This gives

$$
\begin{aligned}
P(x) & =\frac{e^{-\frac{1}{2}[x+\eta t / \alpha]^{2} / Q}}{2 \sqrt{2 \pi Q}}\left[1-\operatorname{erf}\left(\frac{R[x+\eta t / \alpha]}{\sqrt{2 Q\left(Q-R^{2}\right)}}\right)\right] \\
& +\frac{e^{-\frac{1}{2}[x-\eta t / \alpha]^{2} / Q}}{2 \sqrt{2 \pi Q}}\left[1+\operatorname{erf}\left(\frac{R[x-\eta t / \alpha]}{\sqrt{2 Q\left(Q-R^{2}\right)}}\right)\right]
\end{aligned}
$$

\subsection{Comparison with Exact Results and Simulations}

Only for the (simple) Hebbian rule can our dynamic order parameters in fact be calculated directly from the microscopic learning rules, even for finite $\alpha$, which provides an excellent benchmark for candidate general theories. Exact evaluation for on-line learning is found to give (Rae et al, 1998):

$$
\begin{gathered}
R=R_{0}+\eta t \sqrt{\frac{2}{\pi}} \quad Q=Q_{0}+\eta t\left[\eta+2 R_{0} \sqrt{\frac{2}{\pi}}\right]+\eta^{2} t^{2}\left[\frac{1}{\alpha}+\frac{2}{\pi}\right] \\
P[x \mid y]=\int \frac{d \hat{x}}{2 \pi} e^{-\frac{1}{2} \hat{x}^{2}\left[Q-R^{2}\right]+i \hat{x}[x-y R]+\frac{t}{\alpha}\left[e^{-i \eta \hat{x} \operatorname{sgn}(y)}-1\right]}
\end{gathered}
$$




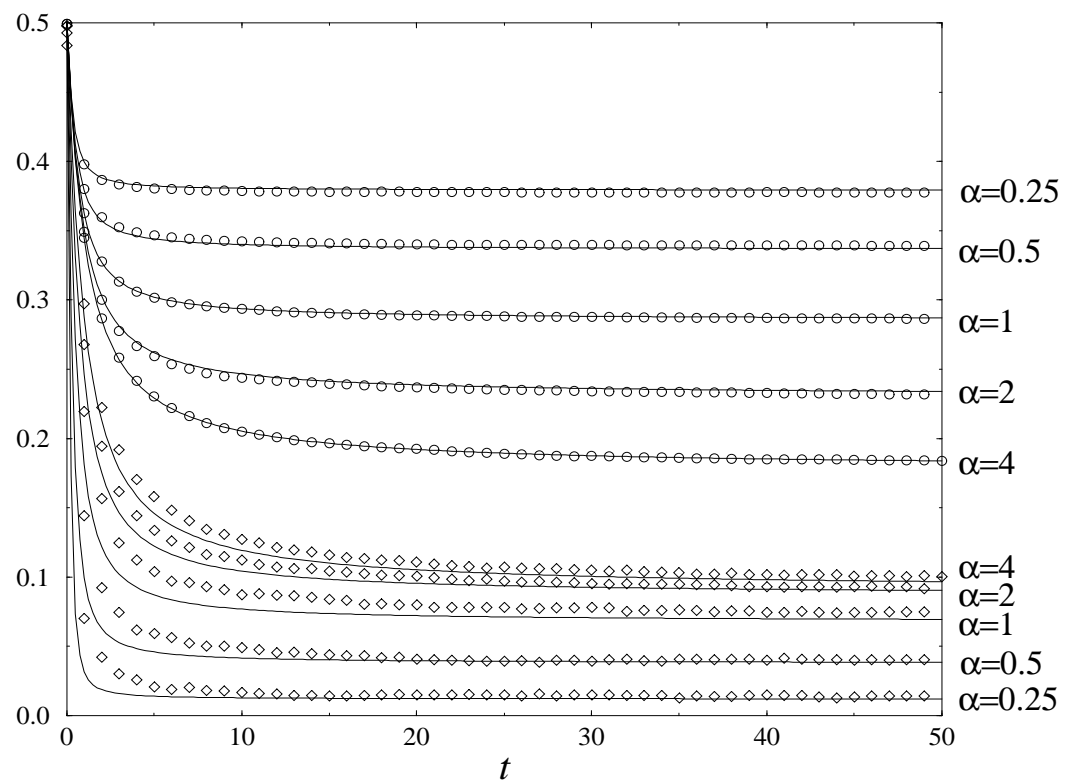

Figure 5: Fig. 5: Comparison between simulation results for on-line Hebbian learning (with system size $N=10,000$ ) and dynamical replica theory, for $\alpha \in\{0.25,0.5,1.0,2.0,4.0\}$. Upper five curves: generalization errors as functions of time. Lower five curves: training errors as functions of time. Circles: simulation results for generalization errors; diamonds: simulation results for training errors. Solid lines: corresponding theoretical predictions.

Comparison with (5.16,5.19) shows that our theory gives the exact expressions for $Q$ and $R$ (and thus for $E_{\mathrm{g}}$ ), but an approximation for $P[x \mid y]$ (and thus for $E_{\mathrm{t}}$ ) as soon as both $\alpha$ and $t$ are finite. The most transparent comparison is made in terms of the Fourier transform $\hat{P}[k \mid y]=\int d x e^{-i k x} P[x \mid y]$ :

$$
\begin{gathered}
\hat{P}[k \mid y]_{\text {exact }}=e^{-\frac{1}{2} k^{2}\left(Q-R^{2}\right)-i k R y+\frac{t}{\alpha}\left[e^{-i \eta k \operatorname{sgn}(y)}-1\right]} \\
\hat{P}[k \mid y]_{\text {DRT }}=e^{-\frac{1}{2} k^{2}\left(Q-R^{2}\right)-i k R y+\frac{t}{\alpha}[-i \eta k \operatorname{sgn}(y)]}
\end{gathered}
$$

One obtains $\hat{P}[k \mid y]_{\text {DRT }}$ by retaining only the first two orders in the expansion of the term $e^{-i \eta k \operatorname{sgn}(y)}$ in the exponent of $\hat{P}[k \mid y]_{\text {exact }}$. The difference between the exponents of (5.24) and (5.25) is in $\mathcal{O}(t)$ rather than $\mathcal{O}\left(t^{2}\right)$ terms. Since the latter control the asymptotics, exactness of our theory is (for any $\alpha$ ) restored for $t \rightarrow \infty$; thus the asymptotic errors shown in Fig. 4 are also exact.

We conclude: either replica symmetry must be broken (RSB), or our set of order parameters $\{Q, R, P\}$ does not yet obey closed deterministic and self-averaging dynamical equations (otherwise our theory would have been exact, by construction). The natural ways to try improve our theory are thus either to construct RSB solutions of the saddle-point equations, or to add observables to the order parameter set, such as the Green's function (3.6). 

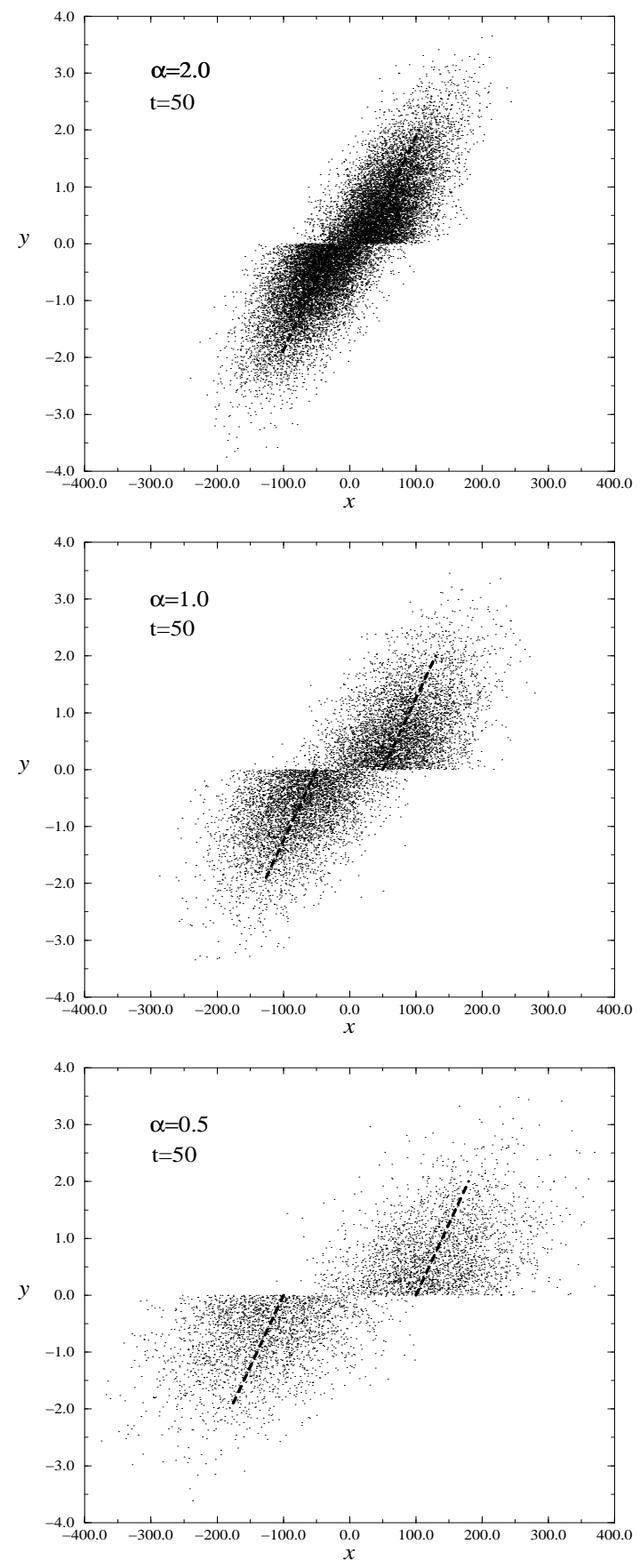

Figure 6: Fig. 6: Comparison between simulation results for on-line Hebbian learning (system size $N=10,000$ ) and dynamical replica theory, for $\alpha \in$ $\{0.5,1.0,2.0\}$. Dots: local fields $(x, y)=(\boldsymbol{J} \cdot \boldsymbol{\xi}, \boldsymbol{B} \cdot \boldsymbol{\xi})$ (calculated for questions in the training set), at time $t=50$. Dashed lines: conditional average of student field $x$ as a function of $y$, as predicted by the theory, $\bar{x}(y)=R y+(\eta t / \alpha) \operatorname{sgn}(y)$. 



Figure 7: Fig. 7: Simulations of Hebbian on-line learning with $N=10,000$. Histograms: student field distributions measured at $t=10$ and $t=20$. Lines: theoretical predictions for student field distributions. $\alpha=4$ (upper), $\alpha=1$ (middle), $\alpha=0.25$ (lower). 
In Fig. 5 we compare the predictions for the generalization and training errors (5.20) of our theory with the results obtained from numerical simulations for $N=10,000$ (initial state: $Q_{0}=1, R_{0}=0$; learning rate: $\eta=1$ ). The curves for the generalization errors show full agreement between theory and experiment, as guaranteed by (5.22). However, the transients of $E_{\mathrm{t}}$ show deviations, which become more pronounced with decreasing $\alpha$, but which vanish with increasing time. This trend immediately follows from (5.24,5.25).

We also compare the theoretical predictions made for the distribution $P[x \mid y]$ with the results of numerical simulations. This is done in Fig. 6, where we show the fields as observed at time $t=50$ in simulations $(N=10,000$, $\eta=1, R_{0}=0, Q_{0}=1$ ) of on-line Hebbian learning, for three different values of $\alpha$. In the same figure we draw (as dashed lines) the theoretical prediction for the $y$-dependent average of the conditional $x$-distribution $P[x \mid y]$ :

$$
\bar{x}(y)=R y+\operatorname{sgn}(y) \eta t / \alpha
$$

We observe that our expression (5.19) for $P[x \mid y]$ (a Gaussian distribution in $x$, with $y$-dependent average given by $\bar{x}(y)$ as given above) indeed captures the qualitative features of the $(x, y)$ statistics. Clearly, $P[x, y]$ is itself not a joint Gaussian distribution.

Finally we compare the student field distribution $P(x)$, as observed in simulations of on-line Hebbian learning $\left(N=10,000, \eta=1, R_{0}=0, Q_{0}=1\right)$ with our prediction (5.21). The result is shown in Fig. 7, for $\alpha \in\{4,1,0.25\}$. The agreement is again quite satisfactory, as could have been expected.

\section{Discussion}

In this paper we have shown how the formalism of dynamical replica theory (e.g. Coolen et al, 1996) can be used successfully to build a general theory with which to predict the evolution of the relevant macroscopic performance measures for supervised (on-line and batch) learning in layered neural networks with randomly composed but restricted training sets (i.e. for finite $\alpha=p / N)$, where the student fields are no longer described by Gaussian distributions, and where the more traditional and familiar statistical mechanical formalism consequently breaks down. For simplicity and transparency we have restricted ourselves to single-layer systems and realizable tasks. In our approach the joint field distribution $P[x, y]$ for student and teacher fields is itself taken to be a dynamical order parameter, in addition to the more conventional observables $Q$ and $R$; from this order parameter set $\{Q, R, P\}$, in turn, immediately follow the generalization error $E_{\mathrm{g}}$ and the training error $E_{\mathrm{t}}$. This then results, following the prescriptions of dynamical replica theory巳,

\footnotetext{
${ }^{2}$ The reason why replicas are inevitable (unless we are willing to pay the price of having observables with two time arguments, and turn to path integrals) is the necessity for finite $\alpha$ to average the macroscopic equations over all possible realizations of the training set.
} 
in a diffusion equation for $P[x, y]$, which we have evaluated by making the replica-symmetric ansatz in the saddle-point equations. This diffusion equation is found to have Gaussian solutions only for $\alpha \rightarrow \infty$; in the latter case we indeed recover correctly from our theory the more familiar formalism of infinite training sets, with (in the $N \rightarrow \infty$ limit) closed equations for $Q$ and $R$ only. For finite $\alpha$ our theory is by construction exact if for $N \rightarrow \infty$ the dynamical order parameters $\{Q, R, P\}$ obey closed, deterministic equations, which are self-averaging (i.e. independent of the microscopic realization of the training set). If this is not the case, our theory is an approximation.

We have worked out our general equations explicitly for the special case of Hebbian learning, where the existence of exact results, derived directly from the microscopic equations (even for finite $\alpha$ ), allows us to perform a critical test of our theory ${ }^{\text {}}$. Here we find that our theory does produce correct predictions for the observables $Q, R$ and $E_{\mathrm{g}}$, but an approximation for $P[x, y]$ and $E_{\mathrm{t}}$ if $\alpha$ is finite (although the stationary state predicted is again correct).

The present study clearly represents only a first step, and many extensions, applications and generalizations can and should be made. To name but a few:

(i) Application to Different Learning Rules

So far our theory has only been applied to Hebbian learning, in view of its special status as a rigorous benchmark (Rae et al, 1998). Further application to non-Hebbian learning rules, subsequently to be tested via simulations, is clearly called for. For rules of the type (5.6) the saddle-point equations are still simple; in general one will have to solve functional saddle-point equations at each instance of time, which is a non-trivial numerical exercise.

(ii) Application to Multi-Layer Networks

Our theory generalizes in a natural way to multi-layer networks, provided the number of hidden neurons remains finite. However, as in the infinite training set formalism, the number of observables (and thus the number of saddlepoint equations) will increase significantly.

(iii) Further analysis of saddle-point equations

We still have to determine the uniqueness or otherwise of the solution of our functional saddle-point equation. More ambitious, but not ruled out, are our current attempts to solve the functional saddle-point equation explicitly. If this is impossible, one further alternative to numerical solution would be a variational approach (where upon choosing a restricted parametrized family of functions, functional extremization is replaced by ordinary extremization).

\section{(iv) Replica Symmetry Breaking}

The observed deviations between the present theory and the exact calcula-

\footnotetext{
${ }^{3}$ Note that such exact results can only be obtained for the relatively simple Hebbian rules, where the dependence of the weight updates $\Delta \boldsymbol{J}(t)$ on the current weights $\boldsymbol{J}(t)$ is trivial or even absent (a decay term at most), whereas our present theory generates macroscopic equations for arbitrary learning rules.
} 
tion for the Hebb rule could indicate RSB (although this is not likely, in view of the asymptotic exactness of our RS equations). In the usual manner one can calculate an equation for the AT instability, which would in our problem define a surface in order parameter space, and determine the onset of RSB.

(v) Systematic Improvement via Higher Order Observables

Dynamical replica theory allows for systematic improvement. By adding new observables to the order parameter set (which cannot be expressed in terms of those already present) the theory will, by construction, become more accurate. A natural candidate for being added to the set $\{Q, R, P\}$ is the Green's

function $\mathcal{A}\left[x, y ; x^{\prime}, y^{\prime}\right]$ (3.6). This would change our problem to closure of a dynamic equation for $\mathcal{A}$, which would involve a higher order Green's function.

(vi) Generalization to Noisy Teachers

Last but not least, one can generalize our theory to the case of noisy teachers. This is a straightforward although tedious exercise, involving a field distribution of the form $P[x, y, z]$ (describing student fields, fields of the 'perfect' teacher, and fields of the 'noisy' teacher). It will, however, allow us to describe over-fitting phenomena in terms of macroscopic dynamic equations.

\section{Acknowledgement:}

DS acknowledges support by EPSRC Grant GR/L52093.

\section{References}

Barber D., Saad D. and Sollich P. (1996), Europhys. Lett. 34, 151

Biehl M. and Schwarze H. (1992), Europhys. Lett. 20, 733

Biehl M. and Schwarze H. (1995), J. Phys. A: Math. Gen. 28, 643

Coolen A.C.C., Laughton S.N. and Sherrington D. (1996), Phys. Rev. B 53, 8184

Coolen, A.C.C and Saad D. (1998), in preparation.

Horner H. (1992a), Z. Phys. B 86, 291

Horner H. (1992b), Z. Phys. B 87, 371

Kinouchi O. and Caticha N. (1992), J. Phys. A: Math. Gen. 25, 6243

Kinzel W. and Rujan P. (1990), Europhys. Lett. 13, 473

Mace C.W.H. and Coolen A.C.C (1998a), Statistics and Computing, in press

Mace C.W.H. and Coolen A.C.C (1998b), in preparation

Mézard M., Parisi G. and Virasoro M.A. (1987), Spin-Glass Theory and Beyond (Singapore: World Scientific)

Rae H.C., Sollich P. and Coolen A.C.C. (1998), in preparation

Saad D. and Coolen, A.C.C (1998), in preparation

Saad D. and Solla S. (1995), Phys. Rev. Lett. 74, 4337

Sollich P. and Barber D. (1997), to be published in Proc. NIPS*97 